EUROPEAN CENTRAL BANK

ECB WORKSHOP ON THE ANALYSIS OF THE MONEY MARKET

\section{WORKING PAPER SERIES} NO 986 / DECEMBER 2008

\title{
THE TOPOLOGY OF THE
} FEDERAL FUNDS MARKET

by Morten L. Bech and Enghin Atalay

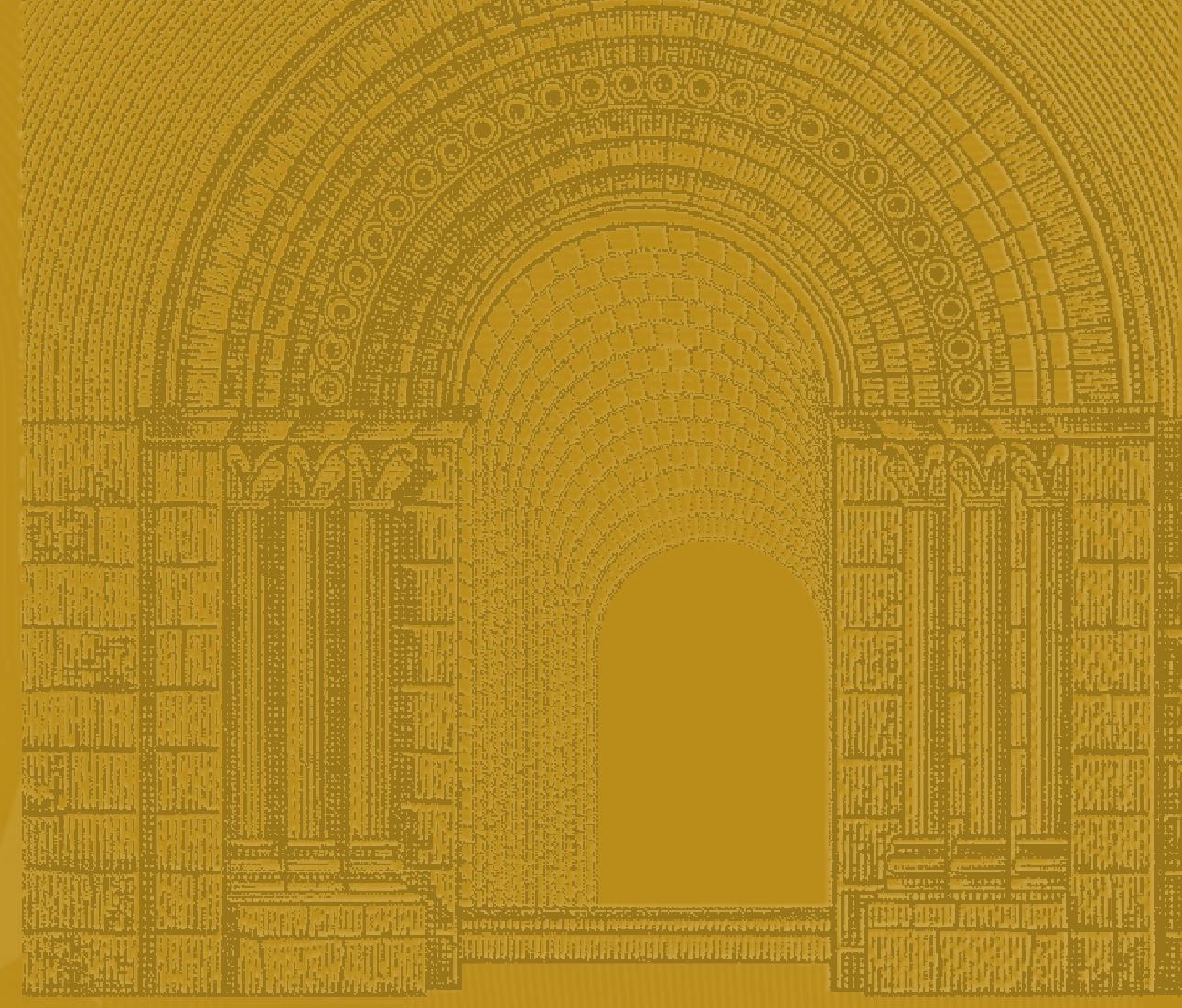




\section{WORKING PAPER SERIES}

NO 986 / DECEMBER 2008

\section{THE TOPOLOGY OF THE FEDERAL FUNDS MARKET'}

by Morten L. Bech ${ }^{2}$ and Enghin Atalay ${ }^{3}$

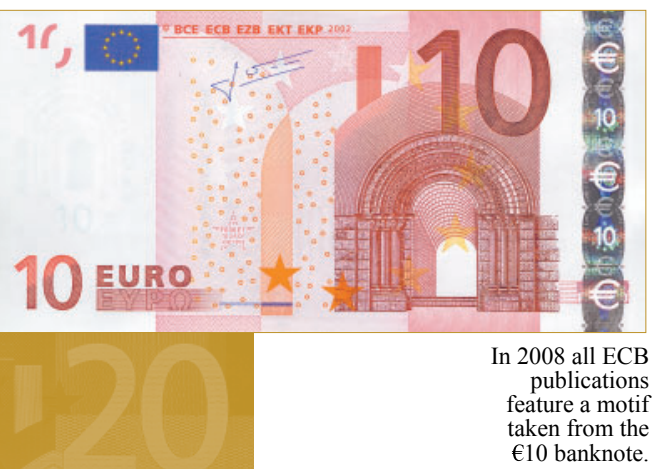

This paper can be downloaded without charge from http://www.ecb.europa.eu or from the Social Science Research Network electronic library at http://ssrn.com/abstract_id $=1299021$.

I The views expressed in this paper do not necessarily reflect those of the Federal Reserve Bank of New York, the Federal Reserve System or the European Central Bank. 2 Federal Reserve Bank of New York, 33 Liberty Street, New York, NY 10045, USA; e-mail: morten.bech@ny.frb.org 


\section{ECB WORKSHOP ON THE ANALYSIS OF THE MONEY MARKET}

On 14 and 15 November 2007, Alain Durré, Huw Pill and Diego Rodriguez-Palenzuela of the ECB's Monetary Policy Stance Division organised a central bank workshop titled "The Analysis of the Money Market: Role, Challenges and Implications from the Monetary Policy Perspective”. This workshop provided an opportunity for participating central bank experts to exchange views and foster debate, also in interaction with international organizations and academic institutions. The first day of the workshop addressed issues related to the macro-perspective of the money market, drawing on the experiences of a large number of countries. The second day adopted a micro-perspective on the money market, looking in particular at trading behaviour in the overnight money market and its implications for the evolution of spreads.

A first version of this paper was presented at this workshop. The papers presented at the time of the workshop did not consider the potential implications of the financial turmoil for the results of the paper, given that the tensions in money markets emerged in August 2007. The published version of these papers represents an update of the original paper, which incorporates the discussion which took place at the workshop and in most cases a discussion on the developments in the money markets since August 2007.

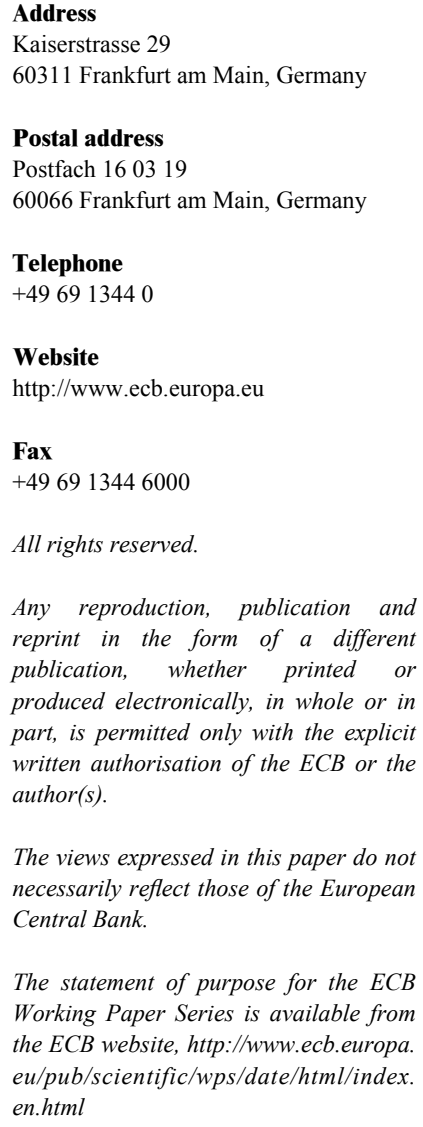




\section{CONTENTS}

Abstract

Non-technical summary

1 Introduction

2 Federal funds market

3 Literature review

4 Data

4.1 Federal funds loans in 2006

4.2 Federal funds loans since 1997

Federal funds networks

6 Visualizing the federal funds network

6.1 The inter-district network

6.2 Intraday network

7 Topological characteristics

7.1 Size, connectivity and reciprocity

7.2 Components

7.3 Degree distribution

7.4 Link weights and node strength

7.5 Degree correlation

7.6 Clustering coefficient

7.7 Distance and diameter

7.8 Node centrality

8 Conclusion

References

Appendices

European Central Bank Working Paper Series 


\begin{abstract}
We explore the network topology of the federal funds market. This market is important for distributing liquidity throughout the financial system and for the implementation of monetary policy. The recent turmoil in global financial markets underscores its importance. We find that the network is sparse, exhibits the small world phenomenon and is disassortative. Reciprocity tracks the federal funds rate and centrality measures are useful predictors of the interest rate of a loan.
\end{abstract}

Keywords: network, topology, interbank, money market

JEL Classification: E4, E58, E59, G1 


\section{Non-technical summary}

We study the overnight interbank money market in the United States -the federal funds market-from 1997 to 2006 through the prism of network topology. That is, we represent the market activity on a given day as a network, where banks are nodes and loans from one bank to another are directed links. Using measures developed to characterize complex networks in the physical sciences, we are able to uncover several salient features of the market.

The simplest measures count the number of nodes or links in each network: in 2006470 banks (nodes) participated, on average, each day and there were, on average, 1543 interbank relationships (links) per day. The federal funds market has been shrinking over time-in terms of the number of active nodes-since 1997. On the other hand, over the sample period, the value settled per day in the federal funds market has increased by $70 \%$, in real terms.

Other measures describe the participants in the federal funds network. In 2006, the average bank lent to 3.3 other institutions. It borrowed, on average, 5.54 loans worth roughly $\$ 719$ million per business day. Relative to 1997, the average bank in 2006 had roughly the same number of counterparties, but conducted more business, in terms of value. These averages disguise the vast heterogeneity that exists among participants. While more than half of the banks in the federal funds market borrowed from at most two other institutions, each day a handful of banks borrowed from a large number of institutions. This phenomenon-where a small number of participants have many relationships and most participants have few-is a characteristic of many other real-world networks.

A third set of measures defines the overall structure of the federal funds market. For instance, in many networks the number of links necessary to reach a particular node from any other node is small. This is also the case for the federal funds network. This tells us that the degree of intermediation between net demanders of federal funds and net suppliers is small. A second measure of the overall structure of a network is used to partition the federal funds market into three groups of banks: 1) banks which supply funds to the market, 2) banks which demand funds from the market, and 3) banks which coordinate supply and demand. Consistent with the literature on the federal funds market, the number of banks in the first group is much larger than the number of banks in the last two groups. The banks in the first group are, in general, smaller and have fewer counterparties than banks in the second or third groups. We are able to conclude that the federal funds market consists of a large number of small banks which are each net lenders to a few large banks.

One additional measure that we use to characterize the federal funds network is the reciprocity of a network. In the context of the federal funds market, reciprocity gives the proportion of bank relationships where both bank $A$ lends to bank $B$ and bank $B$ lends to bank $A$ on a given day. We find that this reciprocity measure used on federal funds network has been tracking the target federal funds rate throughout sample period.

We conclude with an application of a network statistic that is used to determine how central a bank is in the federal funds market. Specifically, we hypothesize that a bank will be able to extract a favorable rate on a loan if it is more central than its counterparty. The hypothesized relationship holds: interest rates are roughly 3 basis points higher when the centrality score of the lender is greater than the centrality score of the borrower. This relationship, while statistically and economically significant, explains only a small fraction of the variability in interest rates. 


\section{Introduction}

The recent turmoil in global financial markets underscores the continuing importance of the federal funds market. Its smooth functioning is of the utmost importance for distributing liquidity throughout the financial system and for the practical implementation of monetary policy. Banks rely heavily on the federal funds market to offset liquidity shocks and manage their reserve positions over the maintenance period. In formulating monetary policy, the Federal Open Market Committee (FOMC) sets a target for the effective federal funds rate and directs the Federal Reserve Bank of New York to "create conditions in reserve markets" that will encourage federal funds to trade near the target. Creating such conditions became a significant challenge beginning in the month of August 2007. Massive amounts of liquidity were injected into the market on several occasions. Nevertheless, the federal funds rate deviated from its target considerably more than usual both within and across days.

In this paper, we take an in-depth look at the structure of the federal funds market from the vantage point of network topology. Networks have proven to be useful in analyzing a wide array of structures and interactions across a multitude of fields. In recent years, the physics community has made significant progress towards understanding the structure and functioning of complex networks. The literature has focused on characterizing the structure of networked systems and how the properties of the observed topologies relate to stability, resiliency and efficiency in case of perturbations and disturbances. Recently, economists have started to show (renewed) interest in networks.

We represent the federal funds market as a network, where financial institutions are nodes and loans are directed links, weighted by the value of the loans between the counterparties. Using a unique transactionlevel data set spanning 1997 to 2006, we are able to analyze in unprecedented detail the characteristics of the overnight federal funds network and its evolution over the past decade. We find that the value sent between banks has increased even though the number of participants of the network has decreased. Like other complex networks, the federal funds network is sparse, exhibits the small world phenomenon, and is disassortative. The number of counterparties per bank (degree) follows a fat-tailed distribution, with most banks having few counterparties and a small number having many. However, unlike other networks, the degree distribution is not necessarily best represented by a scale free distribution. Using network specific measures, we are able to shed new light on the small-bank large-bank dichotomy of the federal funds market, whereby small banks generally lend funds to larger banks. Finally, we provide preliminary evidence that centrality measures, which determine the relative importance of banks in the network, are useful predictors of the interest charged between banks.

In Section 2, we begin with a quick overview of some of the institutional details of the federal funds market. In the subsequent section we review literature on the federal funds market and network theory. Section 4 describes the data used in our study. Section 5 outlines some network terminology. Section 6 presents different ways to visualize the federal funds network. Section 7 contains the analysis and results of the paper. Section 8 concludes.

\section{Federal funds market}

The federal funds market is the market for immediately available reserve balances at the Federal Reserve. Depository institutions that maintain accounts at the Federal Reserve can borrow (buy) or lend (sell) reserve balances. Federal funds, or fed funds, are unsecured loans and the rate at which these transactions occur is called the fed funds rate. Most trades are delivered on the same day and the duration is typically overnight, but trades for longer terms (called term fed funds) also take place. In this paper we consider only overnight fed funds.

Depository institutions hold reserve balances at the Federal Reserve Banks to meet reserve requirements - an average over a two week period - and to prevent any overnight overdrafts that may arise from their payment activities with other depository institutions. ${ }^{1}$ The day to day flows of business to and from a bank are unlikely to leave it with the desired level of reserves. The federal funds market is one option to adjust

\footnotetext{
${ }^{1}$ The Federal Reserve's Regulation D requires banks operating in the United States to hold reserves, either in the form of balances in deposit accounts at the Federal Reserve or as cash in their vaults, in a fixed proportion to some of their deposit liabilities.
} 
the level of reserves. A bank with a shortfall may buy federal funds. Like bank deposits, borrowed federal funds are bank liabilities. From a regulatory point of view borrowed fed funds are treated differently and are not subject to reserve requirements. ${ }^{2}$

There are two methods for buying and selling federal funds. Depository institutions can either trade directly with each other or use the services of a broker. Federal funds brokers do not take positions themselves but rather bring buyers and sellers together on an anonymous basis. In the direct trading segment, transactions commonly consist of sales by small-to-medium sized banks to larger banks and often take place on a recurring basis. The rate is set in reference to the prevailing rate in the brokered market. In the brokered segment participation is mostly confined to larger banks acting on their own or a customer's behalf. Trade sizes are generally larger than in the direct trading segment (see Stigum (1990) ).

There are two ways to settle federal funds trades. Participants can use the Fedwire Funds Service to transfer reserves from one depository institution's account to another. The other method is correspondent re-bookings, where mostly smaller banks that do not have accounts at the Federal Reserve may have their deposits at larger correspondents re-booked as overnight loans to those correspondents. The re-booking segment does not involve wire transfers between accounts at the Federal Reserve. The individual loans are typically smaller and the interest rates tend to be $1 / 4$ percentage point below the rates in the brokered market (see Demiralp et al. (2004)).

By facilitating the transfer of the most liquid funds among depository institutions, the fed funds market plays a major role in the execution of monetary policy. In fact, the directive for implementation of U.S. monetary policy from the FOMC to the Federal Reserve Bank of New York states that the trading desk should "create conditions in reserve markets" that will encourage fed funds to trade at a particular level. Using open market operations the desk can change the supply of reserve balances and thus create upward or downward pressure on the fed funds rate.

\section{Literature review}

The importance of the federal funds market has not escaped the notice of academic research. Ho \& Saunders (1985) develop a model where banks have a target reserve balance that they achieve via participation in the federal funds market. Banks that are risk averse to having a deficient balance will have interest-rate-elastic demand functions for purchasing federal funds. Since large banks have more options to obtain necessary reserves, they are less risk averse. Because of this, large banks end up as net borrowers, while small banks are net lenders in the federal funds market. Allen \& Saunders (1986) provide a second explanation for the large bank-small bank dichotomy. Since federal funds loans are uncollateralized, banks face a risk when lending. Also large banks may not be able to correctly judge the risk of lending to small, rural banks. Because of this information asymmetry, small banks are either rationed out of the federal funds market or are forced to pay higher interest rates. Allen et al. (1989) empirically confirm these results. They find that banks with an asset size greater than $\$ 2.5$ billion (in 1989 dollars) are more likely to be net borrowers in the federal funds market. Also banks that are located away from the major banking centers are more likely to be net lenders than banks that are in New York, Chicago or San Francisco. In the collateralized repo market, banks' size and location do not have the same constraining effects. Furfine (1999) uses transaction level data to describe the market microstructure of the federal funds market by categorizing banks by asset size. His characterization of the fed funds market largely agrees with that of Allen et al. (1989). However, while smaller banks are usually net lenders and larger banks are net borrowers, most banks are active on both sides of the market. We add to this literature by examining how the size and position of a bank in the federal funds market affects their borrowing and lending interest rates.

Hamilton (1996) shows that the effective daily federal funds rate does not follow a martingale. Rates are lower on Fridays, and generally decrease throughout the first seven days of the reserve maintenance period. Demiralp et al. (2004) use a data set similar to the one used by Furfine (1999) to look for possible calendar effects on the interest rate charged on federal funds loans. Interest rate volatility increases during the last three days of the maintenance period. They confirm Hamilton's observation of lower than average interest rates on Friday, but find no evidence of decreasing interest rates during the first seven days of the maintenance

\footnotetext{
${ }^{2}$ Regulation D exempts from the definition of a "deposit," and hence from reserve requirements, bank liabilities arising from borrowings from other banks, from various government agencies and, under certain conditions, from securities dealers.
} 
period. Bartolini et al. (2005b) use a dataset of brokered transactions to study the intraday behavior of the federal funds market. While average interest rates are only slightly lower in the late afternoon, the volatility of interest rates is much higher in this period.

Complex networks drawn from vastly different real world settings tend to exhibit a number of universal properties (see Newman (2003) and Albert \& Barabási (2002)). First, the probability that a node in the network is connected to $x$ other nodes follows a scale-free distribution, $P(x) \backsim x^{-\gamma}$ with $2 \lesssim \gamma \lesssim 3$. This implies that, while most nodes have only few connections, "highly connected hubs" can exist. Second, the probability that the two nearest neighbors of a node are connected (known as clustering) is higher than what would be found in a network where nodes are connected at random. Third, many networks exhibit the small world property, which implies that any node can be reached from any other node in only a few steps. Fourth, certain networks, e.g. social networks, tend to be assortative in that nodes tend to connect with nodes of similar degree while other networks, such as technological and biological networks, tend to be disassortative.

Albert et al. (2000) show that the distribution of the number of counterparties of a financial market participant has important consequences with respect to the resiliency of the network. Networks where the number of counterparties of a participant follows a power law distribution are robust to the removal of random nodes from the network. However, if nodes that are connected to many other participants are removed, the structure of the network can change dramatically. The structure of the network affects the degree to which financial crises spread throughout the market. Sparse networks are more likely to experience financial contagion (see Allen \& Gale (2000)). Gale \& Kariv (2007) set up an experiment where participants bid for an asset. The price of the asset is affected by the structure of the network. As the network becomes more sparse and intermediation in the network increases, the efficiency from trade decreases. Actual data on transactions in the federal funds market reflect the costs of maintaining interbank relationships. So, observed transactions are a good proxy for potential transactions. This fact, combined with the results of Gale \& Kariv (2007), has implications for our paper, as the federal funds market is far from complete and there is some intermediation.

Our paper closely follows the analysis of Soramäki et al. (2006). The authors use statistics developed in network theory to describe Fedwire, a large value interbank payment system operated by the Federal Reserve. The network of interbank payments consists of a core of highly-connected banks with a great majority of the banks having few counterparties on a particular day. The distribution of the number of counterparties for each bank roughly follows the power law distribution. Boss et al. (2004) use data on bank liabilities to study the Austrian interbank network and also find that the number of counterparties per bank follows the power law distribution.

Similar to other financial markets, we find that, in the federal funds market, participants' location matters. Portes \& Rey (2005) use a gravity model-a model where asset flows between two countries is positively related to the size of the countries and inversely related to the distance between the two countries-to find determinants for equity flows among 14 countries. The authors find that the distance is, indeed, inversely related to the size of equity flows between two countries. Furthermore, Portes and Rey show that including variables that proxy for information frictions between two countries decreases the effect of distance on crossborder flows. Similar to Portes \& Rey (2005), Rosati \& Secola (2005) study cross-border payment flows in two EU large-value payment systems. They find that the payment flows between two countries increase significantly if the two countries share a border or a common language and decrease significantly as the distance between the two countries increases.

\section{Data}

We construct our networks from the transaction journal of the Fedwire Funds Service (Fedwire). ${ }^{3}$ The Fedwire Funds Service provides a real-time gross settlement system in which more than 7,000 participants initiate funds transfers, which are immediate, final, and irrevocable when processed. Participants that maintain a reserve or clearing account with a Federal Reserve Bank may use Fedwire to send payments to,

\footnotetext{
${ }^{3}$ The Federal Reserve also operates the Fedwire Securities Service which is the depository for U.S. Treasury and U.S. government agency securities (including mortgage-backed securities), as well as securities issued by certain international organizations such as the World Bank.
} 
or receive payments from, other account holders directly. Participants use Fedwire to handle large-value, time-critical payments, such as payments for the purchase, sale, and financing of securities transactions; the disbursement or repayment of loans; and the settlement of real estate transactions. In addition, participants also use Fedwire for the settlement of interbank purchases and sales of federal funds. As such, federal funds loans are a subset of the Fedwire transactions. We shall refer to the complement of the federal funds-related Fedwire transactions as Fedwire payments.

We identify federal funds loans from the transaction journal using the method pioneered by Furfine (1999). The basic idea is as follows. A pair of transactions between two depository institutions on consecutive business days is identified as a federal funds loan if the amount transferred on the first day is reversed on the next business day with a plausible amount of interest. Each loan identified provides information on the identity of the lender (seller), the identity of the borrower (purchaser), the amount, the time of delivery, the time of return and the interest paid. For a more detailed description of the data, see Appendix B. As noted by Furfine (1999) there are several caveats to be kept in mind when analyzing the data. First, our algorithm only seeks to identify overnight loans and not term loans. ${ }^{4}$ Second, the time of delivery is the time money was moved across accounts at the Federal Reserve and not the time at which the trade was agreed upon. Third, the method might identify other overnight loan arrangements that are not necessarily federal funds e.g. Eurodollars or tri-party repos. In fact, more than half of the transactions in our dataset could be Eurodollar deposits. In many ways Eurodollar deposits and federal funds loans are part of an integrated money market. For example, Bartolini et al. (2008) have shown that the spread between overnight Eurodollar deposits and overnight fed funds loans is less than half of a basis point. For ease of exposition, we will abuse the terminology and refer to the overnight money market loans in our dataset as federal funds loans. Fourth, although we have the identities of sending and receiving banks on each side of the transaction, these may not be the same as the actual borrower and lender. A bank could be conducting an overnight transaction on behalf of a correspondent bank or a non-financial institution. Fifth, correspondent re-bookings are not included in this dataset.

\subsection{Federal funds loans in 2006}

Our sample consists of a total of 6,889,674 federal funds loans for the 2,454 business days between April 1, 1997 and December 29, 2006. During 2006, an average of 2,607 \pm 135 (standard deviation across days) loans worth $\$ 338 \pm 26$ billion were identified per day. ${ }^{5}$ The peak day of 2006 involved 2,964 loans worth $\$ 421$ billion. Federal funds loans come in many sizes with the most common loan sizes being $\$ 100$ million, $\$ 50$ million, $\$ 25$ million and $\$ 200$ million. In 2006, the largest loan identified was $\$ 5$ billion. The distribution of loan sizes is skewed to the right with the median loan being $\$ 50 \pm \$ 1$ million compared to the average loan of $\$ 130 \pm \$ 7$ million. In comparison, the average transaction over Fedwire is $\$ 5$ million and the median is $\$ 36,000$.

The interest rates charged on the loans were centered narrowly around the federal funds target rate with a daily standard deviation of $6.7 \pm 5.0$ basis points. Only a small percentage of loans $(0.2 \%$, value weighted) had an interest rate that was more than 50 basis points from the federal funds target rate.

The intraday distributions of federal funds deliveries and returns are both unimodal and heavily concentrated in the late afternoon. ${ }^{6}$ Almost two thirds of all federal funds deliveries occur after 4:30 p.m. ET whereas $50 \%$ of returns do. This is in contrast to the bimodal intraday patterns in federal funds trading activity found by Bartolini et al. (2005b) using data from a fed funds broker. The first peak in trading activity occurs in the morning between 8:30 a.m. and 10:00 a.m. and the second peak, at even higher levels, late in the afternoon. The analysis shows that $40 \%$ of total value traded is settled in the last two hours. So, many of the loans that are arranged in the morning are settled over Fedwire in the late afternoon.

Federal funds are generally considered " 24 hour money" even though a market for early return federal funds does exist. In our data, $14 \%$ of returns occur before noon and the interest paid on these early return

\footnotetext{
${ }^{4}$ Maturities in the "term" federal funds market range from a few days to more than a year, although most transactions mature in six months or less. The amount of term federal funds outstanding is probably on the order of one-tenth of the amount of overnight loans arranged on any given day (Meulendyke (1998)).

${ }^{5}$ A total of 646,423 loans were identified in 2006 .

${ }^{6}$ The Fedwire Funds Service business day begins at 9:00 p.m. Eastern Time (ET) on the preceding calendar day and ends at 6:30 p.m. ET. On December 8, 1997 the Fedwire Funds Service opening time was moved from 07:00 a.m to 00:30 a.m. ET The current opening time was implemented on May 17, 2004. The closing time has remained unchanged.
} 

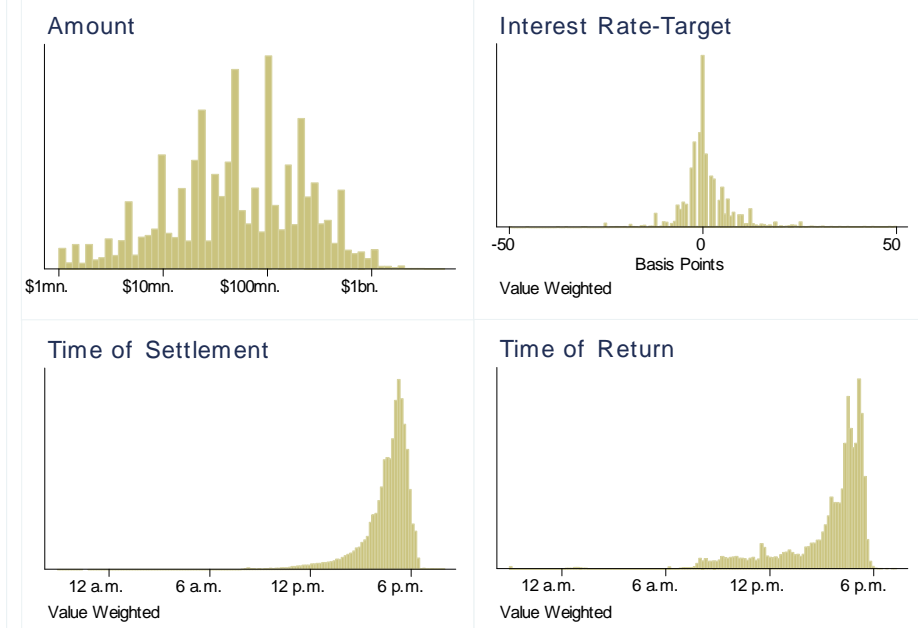

Figure 1: Distribution of size, interest rate, time of settlement and time of return for federal funds loans in 2006.

loans is usually several basis points lower than for loans returned in the afternoon. In 2006, the average (value-weighted) time of delivery was 4:30 p.m. \pm 0:07 minutes while the average time of return was 3:09 p.m. \pm 0:09 minutes - implying an average duration of the loans of 22 hours and 39 minutes.

\subsection{Federal funds loans since 1997}

The overall market turnover has increased by $70 \%$ in real terms from around $\$ 200$ billion in 1998 to $\$ 340$ in $2006 .^{7}$ However, the share of Fedwire transactions being identified as federal funds loans in terms of value has been fairly constant at $15 \%$ over the years (see Figure 2). The number of loans per day has been falling while individual loans have grown larger in size. The number of loans per day has decreased by $23 \%$ from around 3,300 in 1998 to 2,600 in 2006 while the average loan size has doubled from $\$ 60$ million to $\$ 120$ million (see Figure 3). Moreover, loans tend to settle later in the afternoon and have less daily variation in their interest rates. Both the average time of delivery and return have moved approximately half an hour later (see Figure 3). Prior to 2002, the standard deviation of the interest rates charged on a day was $13.8 \pm$ 5.8 basis points, whereas in 2006 the standard deviation was $6.7 \pm 5.0$ basis points.

\section{$5 \quad$ Federal funds networks}

A network is a collection of nodes joined in pairs by directed links. Links can have weights attached, measuring the strength of the relationship between nodes. Mathematically, a weighted directed network, $N$, is defined by a set $\mathcal{V}(N)$ of $n$ nodes (vertices), a set of $\mathcal{E}(N)$ of $m$ links (edges), and a mapping $\omega: \mathcal{E}(N) \longmapsto \mathbb{R}$. Each node is identified by an integer value $i=1, \ldots, n$; the links are identified by a pair $(i, j)$ that represents a connection going from node $i$ to node $j$ to which a weight $\omega(i, j)$ is attached. In principle, self connections, i.e., $(i, i)$ or multiple links, i.e., $\left(i_{1}, j_{1}\right)$ and $\left(i_{2}, j_{2}\right)$ where $i_{1}=i_{2}$ and $j_{1}=j_{2}$, between nodes can exist. If multiple links do not exist, a directed, weighted network can be completely represented in terms of a weight matrix $\boldsymbol{W}$, where each element $w_{i j}=\omega(i, j)$ is the weight on the link from node $i$ to node $j$. At times it can be useful to consider an unweighted version of a network. A convenient way of representing an unweighted network is by using an adjacency matrix, a $n \times n$ matrix denoted by $\boldsymbol{A}$ such that

$$
a_{i j}=\left\{\begin{array}{cc}
1 & \text { if }\left|w_{i j}\right|>\text { threshold } \\
0 & \text { Otherwise }
\end{array}\right.
$$

\footnotetext{
${ }^{7}$ In this paper, all dollar figures are stated in 2007 dollars or in nominal figures.
} 

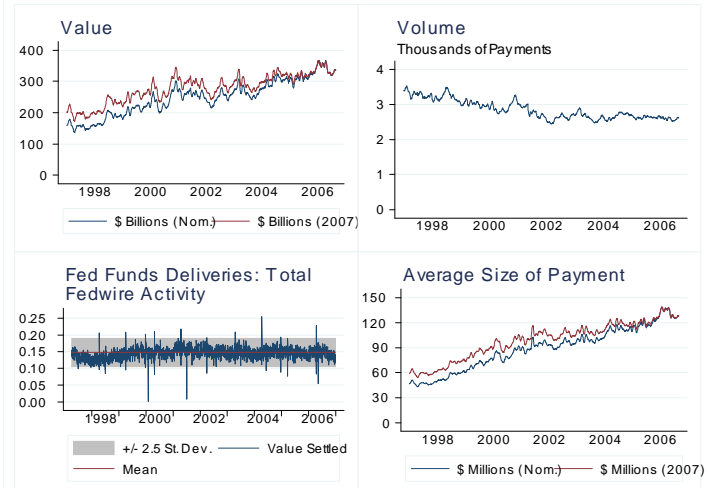

Figure 2: Top left: Total daily value of federal funds loans. Top right: Number of federal funds loans per day. Bottom left: Ratio of value of federal funds deliveries to total activity settled over Fedwire. Bottom right: Average size of federal funds loans. Except for the top left panel of this figure, all time series graphs in this paper are displayed as a four-week moving averages.
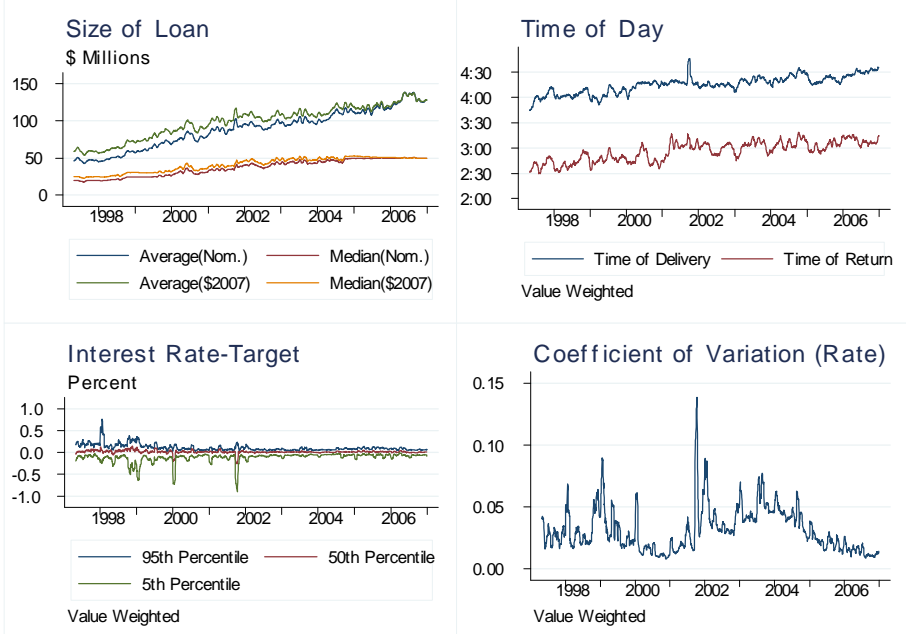

Figure 3: Top left: Median and average loan size. Top right: Mean time of delivery and time of return. Bottom left: 5th, 50th and 95th percentile of difference between the interest rate on fed funds loans and the target interest rate. Bottom right: Coefficient of Variation of the interest rate. 


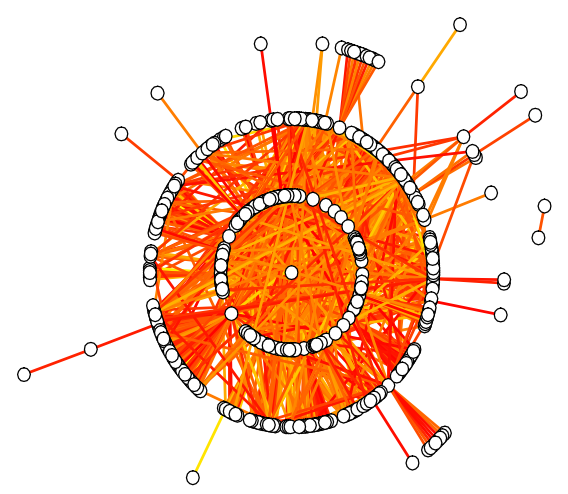

Figure 4: Federal funds network for September 29, 2006. Largest participant-in terms of value-is in the center. Large value links are in yellow; small value links are in red.

where the threshold often is set to zero. The set $\mathcal{P}_{i}=\{j:(j, i) \in \mathcal{E}(N)\}$ is the set of predecessors of node $i$ and $\mathcal{S}_{i}=\{j:(i, j) \in \mathcal{E}(N)\}$ is the set of successors of node $i$. The union of the predecessors and successors is the set of neighbors of node $i, \mathcal{N}_{i}=\mathcal{P}_{i} \cup \mathcal{S}_{i}$.

The set of federal funds loans can be used to form networks in a multitude of ways depending on the definitions of what constitute nodes, links and weights. For most of the analysis, we take the set of nodes to be the institutions which either sent or received federal funds on a particular day. If two banks are counterparties to a loan, then there is a directed link from the lender to the borrower, weighted by the dollar amount of the loan. In the case of multiple loans between two institutions, the weight of the link is the sum of the value of all the loans. Consequently, we have an ensemble of networks across business days.

We also consider an inter-district network. There are twelve nodes in this network, one for each Federal Reserve district. Directed links represent the total daily value that flows between and within the Federal Reserve districts Finally, we look at how the interbank network varies throughout the day. For each day in 2006, we break up the business day into half hour intervals.

\section{Visualizing the federal funds network}

The federal funds loan network for September 29, 2006 - the last business day of the third quarter - is illustrated in Figure 4. A total of 479 banks were active in the market on that day. The largest bank - in terms of total value of federal funds bought and sold - is located at the center of the graph. The first circle consists of the 165 banks with which the bank in the center did business. The second circle consists of the 271 banks that the banks of the first circle did business with. The banks in the second circle had no activity with the bank in the center. The remaining circles represent the 42 banks that were more than two links away from the center. Moreover, two banks did business among themselves but with no other. Large value links are in yellow; small value links are in red.

\subsection{The inter-district network}

Complex networks are generally abstract constructs where the position of the nodes has no particular meaning. In many ways, this is also the case for the federal funds market. Trading takes place on a screen-based system or over the phone and settlement occurs electronically via Fedwire. However, the participants are physically located all over the United States. These participants maintain an account with their local Federal Reserve Bank.

The literature on the federal funds market often mentions the presence of a tiered structure, in which smaller banks will lend to regional banks that lend to money center banks in New York and, to a lesser extent, Chicago. As Stigum (1990) writes “... the federal funds market resembles a river with tributaries: money is collected in many places and then flows through various channels into the New York market. In essence, the nation's smaller banks are the suppliers of fed funds, and the larger banks are the buyers." 


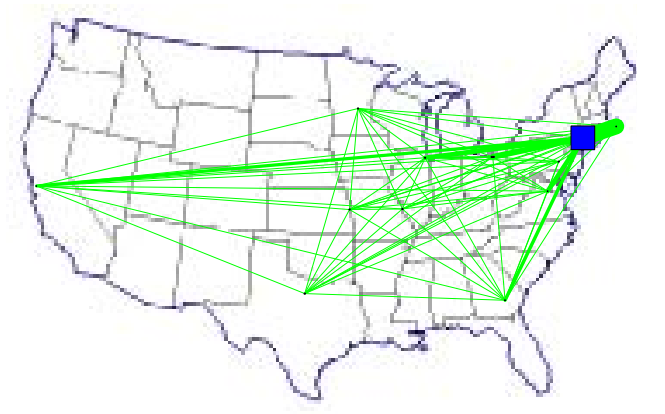

Figure 5: Federal funds Reserve District network on September 29, 2006. The width of each link is proprotional to inter-district activity. The width of each node is proportional to intra-district activity

We construct a Federal Reserve district network by assigning each bank to the district where it resides and then calculating the flows between and within districts. The size of the nodes in Figure 5 scales with the value of loans within the district and the width of the links scales with the value lent and borrowed between districts. Almost a quarter of the participants in the federal funds market were located in the New York district and $79 \%$ of the value involved at least one bank based in this district. The San Francisco, Chicago and Atlanta districts were the next largest districts in terms of number of participants while Boston was the second largest in terms of value. Each district shares a link with a majority of the other districts.

In the spirit of Portes \& Rey (2005), we run the following regression on data from 2006 to test if distance affects the value sent between counterparties.

$$
\log \left(v_{i j}\right)=\theta_{0}+\theta_{1} \log \left(v_{i \cdot}\right)+\theta_{2} \log \left(v_{\cdot j}\right)+\theta_{3} 1(i=j)+\theta_{4} 1(i \text { neighbors } j)+\varepsilon
$$

In this regression, $v_{i j}$ is equal to the value sent from banks in district $i$ to banks in district $j$. The value sent by district $i$ is given by $v_{i}=\sum_{j} v_{i j}$ and the value borrowed by district $\mathrm{j}$ is given by $v_{\cdot j}=\sum_{i} v_{i j}$. The indicator variable $1(i$ neighbors $j)$ is equal to 1 if district $i$ and district $j$ share a border, and 0 otherwise.

As expected, the estimated values of $\theta_{3}$ and $\theta_{4}$ are positive and significant. The value sent between two districts that border each other is nearly $70 \%$ higher than the value sent between districts that do not share a border. The estimated value of $\theta_{3}$ implies that flows within a district are approximately $165 \%$ higher than flows between two non-neighboring districts.

\subsection{Intraday network}

As shown in Figure 1, the settlement activity of federal funds loans is very high during the latter part of the afternoon. This is driven both by trades conducted in the late afternoon and by the settlement of trades that were booked earlier in the day. Another way of illustrating this phenomenon is to create a network for each half hour of the day. Before 3:00 p.m. ET, the network is relatively sparse, but the tiered structure is evident in the first panels of Figure 6. A burst of activity occurs over the next 3 hours until the close for third party transfers (i.e. transfers on the behalf of the bank's customers) at 6:00 p.m. ET. Some residual activity occurs over the last half hour before the close of Fedwire. 


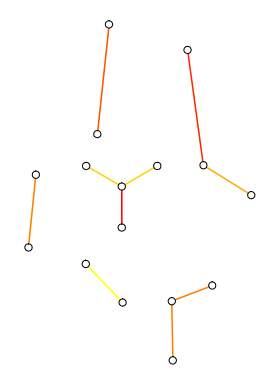

9:01 to 9:30 a.m.

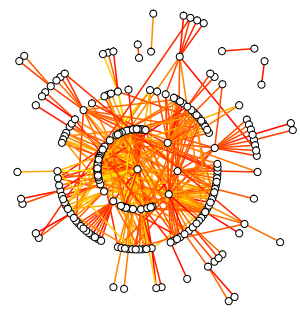

4:31 to 5:00 p.m.

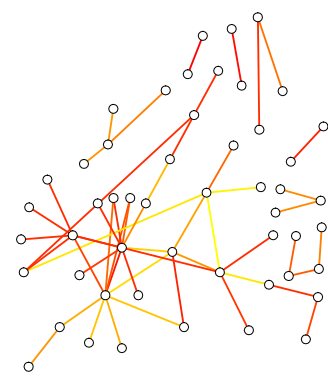

12:01 to $12: 30$ p.m.

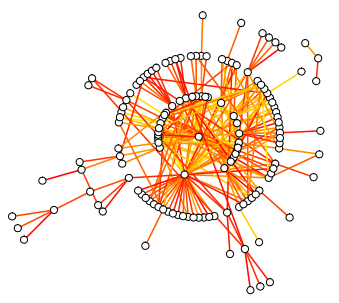

5:01 to 5:30 p.m.

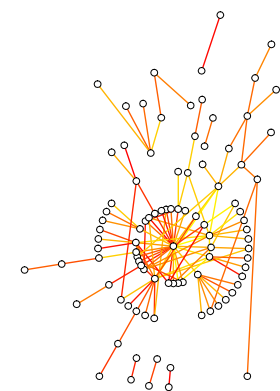

$2: 31$ to $3: 00$ p.m.

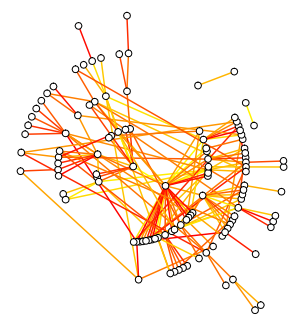

5:31 to $6: 00$ p.m.

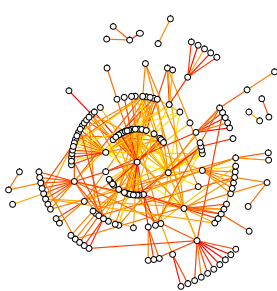

4:01 to 4:30 p.m.

Figure 6: Intraday networks for September 29, 2006. Each network represents the activity in one half-hour period. The center of each network is the same as in Fig. 4. Large value links are in yellow; small value links are in red.

\section{Topological characteristics}

We now turn to a detailed analysis of the topological features of the federal funds network. Our modus operandi is first to define the topological measures and then present the results. In terms of the results, we first focus on the statistics for 2006 and then turn to describing the trends observed since 1997. For the ease of exposition we shall refer to market participants as banks even though institutions such as thrifts and government sponsored enterprises are also important players in the federal funds market. ${ }^{8}$

\subsection{Size, connectivity and reciprocity}

Definition: The most basic properties of a network are the number of nodes, $n$, and the number of links, $m$. The number of nodes defines the size of a network while the number of links relative to the number of possible links, given the number of nodes, is a measure of the degree of completeness of a network. The degree of completeness for a directed network is given by

$$
\alpha=\frac{\sum_{i j} a_{i j}}{n(n-1)}=\frac{m}{n(n-1)}
$$

and ranges from zero for a set of nodes with no links to 1 for a complete network. For a directed network, it is often important to know if its links are reciprocal, i.e., if node $i$ is linked to node $j$, is node $j$ also linked to node $i$ ? The standard way to measure reciprocity is by the fraction of links for which there is a link in

\footnotetext{
${ }^{8}$ As of September 30, 2007 the Federal Home Loan Bank System had sold almost $\$ 100$ billion of (overnight and term) federal funds according to its consolidated balance sheet.
} 
the opposite direction, i.e.,

$$
\varrho=\frac{\sum_{i j} a_{i j} a_{j i}}{m}
$$

However, a potential problem with this measure is that it does not take into account that networks with a higher degree of completeness will tend to have a higher number of reciprocal links due exclusively to random factors (see Costa et al. (2006)). An alternative measure is the correlation coefficient of the adjacency matrix from Eq. 1

$$
\rho=\frac{\sum_{i j}\left(a_{i j}-\alpha\right)\left(a_{j i}-\alpha\right)}{\sum_{i j}\left(a_{i j}-\alpha\right)^{2}}=\frac{\varrho-\alpha}{1-\alpha}
$$

Values of $\rho$ greater than zero imply larger reciprocity than a random network (with the same degree of completeness), while values below zero imply smaller reciprocity than a random network. The former case is referred to as a reciprocal network and the latter as an anti reciprocal network. A weighted version of the standard reciprocity measure can be computed as

$$
\widetilde{\varrho}=\frac{\sum_{i j} a_{i j} a_{j i} w_{i j}}{\sum_{i j} w_{i j}}
$$

and the correlation coefficient for $\mathbf{W}, \widetilde{\rho}$, is obtained by substituting $w_{i j}$ for $a_{i j}$ in the first part of Eq. 5 .

Discussion: In 2006, the average size of the federal funds network on any given day was $470 \pm 15$ nodes. A total of 986 different banks were present in the market on at least one day during 2006 and 157 banks were present on all days. The largest network, consisting of 511 banks, occurred on September 1, 2006. A directed network with 470 nodes implies more than 220,000 possible links. However, only a total of 8,660 different links were identified over the course of 2006. The average number of links was 1,543 \pm 72 links per day with a mere 41 links present on all days. In other the words, the fed funds network is extremely sparse with a degree of completeness averaging only $0.70 \% \pm 0.03 \%$ (see Table 5 ). In this respect the fed funds network is similar to the network of Fedwire payments studied by Soramäki et al. (2006), which also had a degree of completeness of less than $1 \%$.

On the other hand, in terms of reciprocity the two networks are vastly different. The $\varrho$-reciprocity of the federal funds network averaged $6.5 \% \pm 0.8 \%$, which is significantly lower than the reciprocity of the network of Fedwire payments. This is a reflection of the fact that banks are much more likely to send each other payments than grant each other overnight loans on a given day. Moreover, the fed funds network is slightly more reciprocal than a random network with a $\rho$-reciprocity of $5.8 \% \pm 0.8 \%$. The weighted reciprocity measures are $43 \% \pm 3.2 \%$ and $7.0 \% \pm 1.7 \%$ for $\widetilde{\varrho}$ and $\widetilde{\rho}$, respectively (see Table 5 ). Hence, larger value links are more likely to have a link in the opposite direction, everything else being equal.

The federal funds network has shown a steady downward trend in both the number of nodes and links from 1997 to 2006. The average number of participants per day has dropped by more than a quarter from 650 to 470 while the number of directed links per day has dropped by $30 \%$ from 2, 200 to less than 1,600. The degree of completeness has increased marginally from $0.5 \%$ to $0.7 \%$ (see Figure 7 ).

An intriguing empirical finding is that reciprocity has been tracking (or perhaps even been leading) the level of the federal funds rate over most of the period as illustrated in Figure 8. The economic significance (if any) of this observation is not well understood and is a topic for future research. The relationship breaks down for the weighted reciprocity measures.

\subsection{Components}

In analyzing the microstructure of markets it is often useful to categorize participants to gather further insights. Furfine (1999) groups institutions by asset size. ${ }^{9}$ Network topology can also be used to define categories. Here, we employ a methodology proposed by Dorogovtsev et al. (2001), where nodes are categorized according to how they connect with other nodes. The categorization provides a useful decomposition of the market into a core that intermediates funds and components that primarily supply or demand funds. We correlate the network categorization with attributes such as assets and geographical location.

\footnotetext{
${ }^{9}$ Banks with assets less than $\$ 250$ million are grouped together. The other groupings are $\$ 250$ million to $\$ 1$ billion, $\$ 1$ billion to $\$ 10$ billion, $\$ 10$ billion to $\$ 100$ billion, and greater than $\$ 100$ billion.
} 

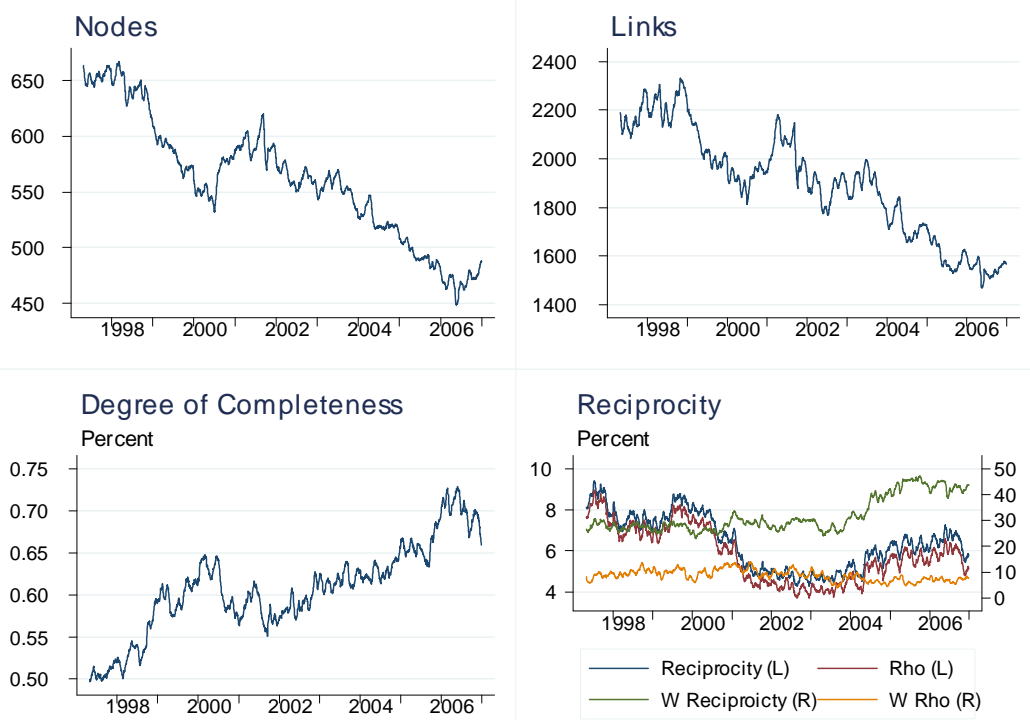

Figure 7: Top left: number of nodes per day in each network. Top right: number of links per day. Bottom left: degree of completeness. Bottom right: reciprocity. Values for the weighted reciprocity measures are plotted along the right axis, while values for the unweighted measures are given on the left axis.

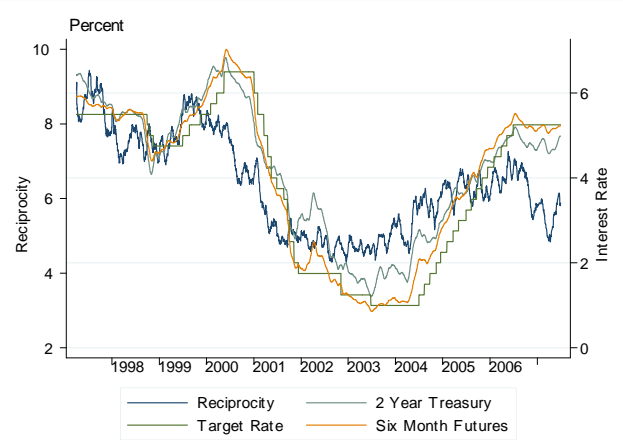

Figure 8: Reciprocity, target federal funds rate, interest rate on the 2 year treasury note and implied 6-month federal funds futures, for April 1, 1997 to July 31, 2007. 


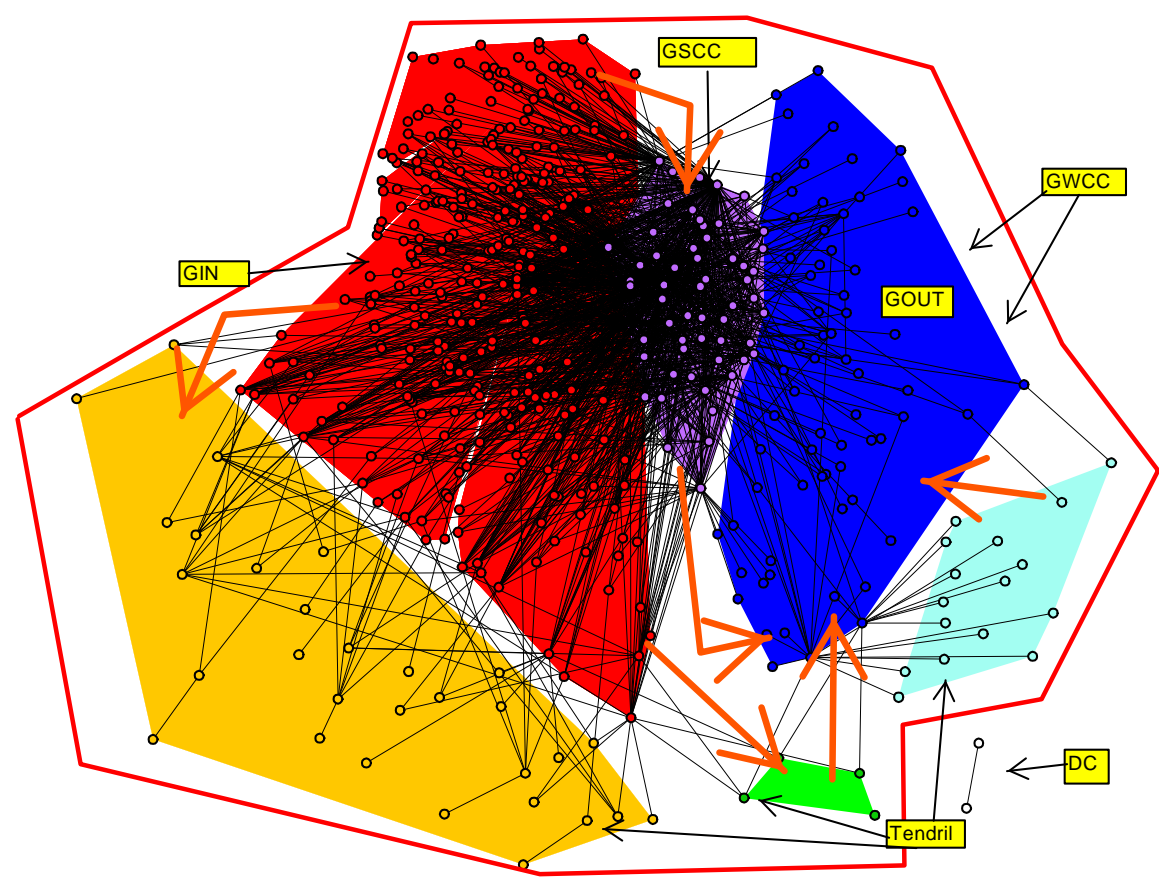

Figure 9: Federal funds network for September 29, 2006. GWCC = giant weakly connected component, $\mathrm{DC}=$ disconnected component, GSCC = giant strongly connected component, GIN = giant in-component, GOUT $=$ giant out- component. On this day there were 57 nodes in the GSCC, 303 nodes in the GIN, 67 nodes in GOUT, 50 nodes in the tendrils and 2 nodes in a disconnected component.

Definition: The nodes of a network can be partitioned into a collection of disjoint sets called disconnected components, $\mathcal{V}(N)=\cup_{h} \mathcal{V}_{h}(N)$. The nodes within each disconnected component do not have links to or from nodes in any other component, i.e., $\left\{(i, j),(j, i): i \in \mathcal{V}_{h}(N) \wedge j \in \mathcal{V}_{h^{\prime}}(N)\right\}=\emptyset$ if $h \neq h^{\prime}$. The component with the largest number of nodes is referred to as the giant weakly connected component (GWCC). In other words, the GWCC is the largest component of the network in which all nodes connect to each other via undirected paths. The remaining disconnected components (DCs) are smaller components for which the same is true. In empirical studies the GWCC is often found to be several orders of magnitude larger than any of the DCs (see Broder et al. (2000)).

The GWCC consists of a giant strongly connected component (GSCC), a giant out-component (GOUT), a giant in-component (GIN) and tendrils (see Figure 9). The GSCC comprises all nodes that can reach every other node in the GSCC through a directed path. A node is in the GOUT if it has a path from the GSCC but not to the GSCC. In contrast, a node is in the GIN if it has a path to the GSCC but not from it. A node is in a tendril if it does not reside on a directed path to or from the GSCC. ${ }^{10}$

Discussion: In the network of payments sent over Fedwire analyzed by Soramäki et al. (2006), the GSCC is the largest component. On average, almost $80 \%$ of the nodes in that network belong to the GSCC. In contrast, the GSCC is much smaller for the federal funds network. In 2006 , only $10 \% \pm 1 \%$ of the nodes belong to this component. By far the largest component is the GIN. In $2006,58 \% \pm 5 \%$ of the nodes were in this component. The GOUT contained $17 \% \pm 4 \%$ of all nodes per day, while there were $14 \% \pm 3 \%$ of the nodes located in the tendrils. ${ }^{11}$ Less than $2 \% \pm 1 \%$ of the nodes were in the remaining disconnected components (see Table 5).

\footnotetext{
${ }^{10}$ The tendrils may also be differentiated into three subcomponents: a set of nodes that are on a path emanating from GIN, a set of nodes that are on a path leading to GOUT, and a set of nodes that are on a path that begins in GIN and ends in GOUT.

${ }^{11} 7.2 \%$ of nodes were in "from-GIN" tendrils, $4.8 \%$ of nodes were in the "to-GOUT" tendrils and $2.3 \%$ of nodes were in "tubes" from GIN to GOUT.
} 


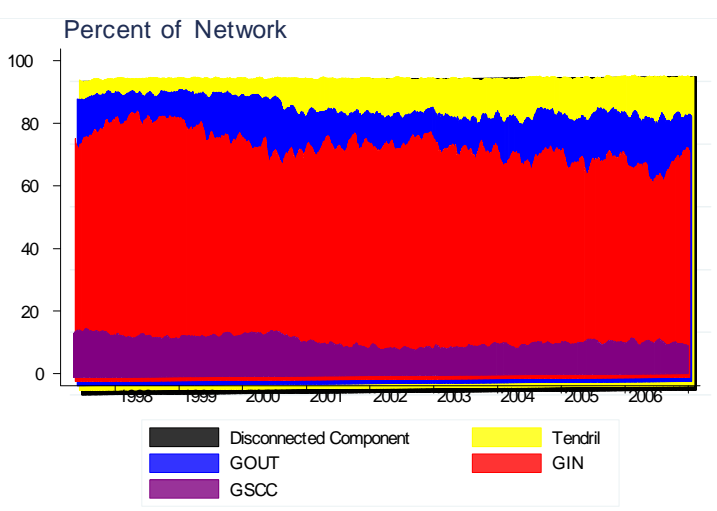

Figure 10: Percentage of nodes in each component, from April 1, 1997 to December 31, 2006.

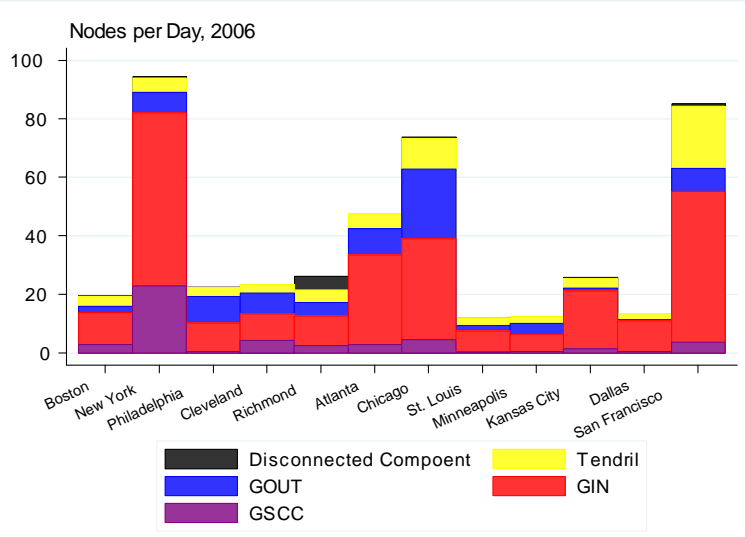

Figure 11: Average number of nodes in each district and each component for each day in 2006. 
The GSCC can be thought of as the core of the market; funds flow among all the banks in that component. Nodes in the GIN were net senders of federal funds while nodes in the GOUT were net receivers. In 2006, only $1.2 \%$ nodes in the GOUT had a higher value of sends than receipts and $99.2 \%$ of the GIN nodes sent more than they received. In other words, banks in the GIN are supplying funds to the core of the market whereas banks in the GOUT are demanding funds from the core. As nodes in the GIN make up nearly $60 \%$ of the nodes in the federal funds network, a majority of banks are net providers of funds to the market. The reserves from these banks are passed on to a small number of banks in the core of the market, which then distribute these funds among themselves and to those banks that demand funds.

Banks in the New York district were overrepresented in the GSCC. In 2006, $49 \%$ of the banks in the GSCC were from the New York district. No other district accounted for more than $10 \%$ of the GSCC. The nodes in the St. Louis, Minneapolis, Kansas City and Dallas districts tended to be in the GIN whereas the share of banks in the GOUT was larger than average in the Chicago district and the Cleveland district (see Figure 11). The nodes in the tendrils are interesting in that they do not receive (supply) their federal funds from (to) the core. Nodes from the San Francisco district are overrepresented in the tendrils.

The relative sizes of the components has been fairly constant throughout the period, even though tendrils have become slightly more prominent at the expense of the GSCC (see Figure 10).

Banks in the GSCC are approximately 20 times larger, in terms of assets, than banks in other components. In 2006, the average bank in the GSCC had an asset size of $\$ 359$ billion. For the GIN, GOUT, tendril and DC these averages were $\$ 10.0$ billion, $\$ 39.6$ billion, $\$ 8.7$ billion, and $\$ 2.1$ billion, respectively.

Membership of a node in a particular component is persistent across time. The probability of a node being in component $\mathcal{C}_{t}$ in on a particular day, given that the node was in component $\mathcal{C}_{t-1}$ on the previous business day is shown in Table 1. The probability of a node belonging to the same component that it belonged to on the previous business day is high, especially for the GIN. Conversely, the probability of transition from GOUT to GIN or from GIN to GOUT is very low. So, if a bank is on the sending or receiving side of the market, it is rather unlikely that the bank will be on the opposite side of the market on the following day. While banks that are in the center of the federal funds market are very likely to participate in the federal funds market on the following business day, banks at the periphery of the market are much less likely to either lend or borrow on the next day.

\begin{tabular}{crrrrrrr}
\hline $\mathbb{P}\left(i \in \mathcal{C}_{t} \mid i \in \mathcal{C}_{t-1}\right)$ & & \multicolumn{7}{c}{$\mathcal{C}_{t}$} & & \\
& GSCC & GIN & GOUT & Tendril & DC & $i \notin \mathcal{V}(N)$ & Total \\
\hline $\mathcal{C}_{t-1}$ & & & & $\%$ & & & \\
GSCC & 76 & 10 & 11 & 1 & 0 & 1 & 100 \\
GIN & 2 & 83 & 1 & 5 & 0 & 9 & 100 \\
GOUT & 7 & 4 & 68 & 9 & 0 & 12 & 100 \\
Tendril & 1 & 20 & 10 & 57 & 1 & 12 & 100 \\
DC & 0 & 15 & 3 & 5 & 46 & 32 & 100 \\
$i \notin \mathcal{V}(N)$ & 0 & 5 & 2 & 2 & 0 & 91 & 100 \\
\hline
\end{tabular}

Table 1: Probability transition matrix. Entries of the matrix give the probability of moving between components on consecutive business days. Only data from 2006 were used for this table.

\subsection{Degree distribution}

The number of counterparties is a key characteristic, in terms of assessing the importance of different market participants. In the federal funds market, the number of counterparties a bank lends to and the number of counterparties it borrows from are of particular interest. In the network literature the number of connections of a node is known as its degree. In the case of a directed network, there are two kinds of degree: out-degree and in-degree.

Definition: The in-degree of a node is the number of its predecessors, $k_{i}^{i n}=\left|\mathcal{P}_{i}\right|=\sum_{j} a_{j i}$. The outdegree of a node is the number of its successors, $k_{i}^{\text {out }}=\left|\mathcal{S}_{i}\right|=\sum_{j} a_{i j}$. For any network, the average in-degree is equal to the average out-degree and also equal to the number of links divided by the number of nodes, i.e., $\left\langle k_{i}^{\text {out }}\right\rangle=\frac{1}{n} \sum k_{i}^{\text {out }}=\left\langle k_{i}^{\text {in }}\right\rangle=\frac{1}{n} \sum k_{i}^{\text {in }}=\frac{m}{n}$. The distribution of degrees across nodes is a basic characteristic of networks and is often used in comparative analysis of networks. The random network 
model of Erdos \& Renyi (1959) is a popular benchmark. ${ }^{12}$ For this type of network the distribution of node degree follows a Poisson distribution. Many other networks, including several networks representing activity over large value payments systems-such as Fedwire (see Soramäki et al. (2006)) and the Japanese interbank payment system BOJ-NET (see Inaoka et al. (2004)) - follow a power law distribution in the tail, $P(x) \backsim x^{-\gamma}$ for $x>a$.

Discussion: On average banks lent to (or borrowed from) $3.3 \pm 0.1$ other banks in 2006 . The average number of counterparties per bank increased slightly from 1999 to 2003 but has fallen back to its 1997 level. The average number does not reflect the substantial heterogeneity across nodes and components. The maximum in-degree was $127.6 \pm 16.3$, while the maximum out-degree in the network was $48.8 \pm 6.4$. So, there are a large number of banks that lend or borrow from only a few counterparties and a small number of banks which have many counterparties.

The disparity between the maximum in-degree and maximum out-degree in the federal funds network points to the asymmetry present in the federal funds market. There are many more banks that lend to a few institutions, but do not borrow than there are banks that borrow from a few institutions, but do not lend. Because of this, the banks which have many counterparties are able to borrow from more institutions than they are able to lend to.

In the GSCC, the average in- and out-degrees were $19 \pm 2$ and $9 \pm 1$, respectively. For the GIN, the average in-degree was 0.2 and the average out-degree was 4 . In the case of the GOUT the average in-degree was 6 and the average out-degree was 0.2 .

\begin{tabular}{crrrrr}
\hline & GSCC & GIN & GOUT & Tendril & DC \\
\hline$\left\langle k_{i}^{\text {in }}\right\rangle$ & 19.1 & 0.2 & 5.9 & 2.0 & 0.7 \\
$\left\langle k_{i}^{\text {out }}\right\rangle$ & 9.3 & 3.8 & 0.2 & 0.7 & 0.7 \\
$\max k_{i}^{\text {in }}$ & 127.6 & 11.3 & 38.2 & 13.7 & 4.3 \\
$\max k_{i}^{\text {out }}$ & 48.8 & 29.0 & 6.5 & 6.6 & 1.1 \\
\hline
\end{tabular}

Table 2: Average in-degree, average out-degree, maximum in-degree and maximum out-degree for each component. Each entry gives the average value across all networks in 2006.
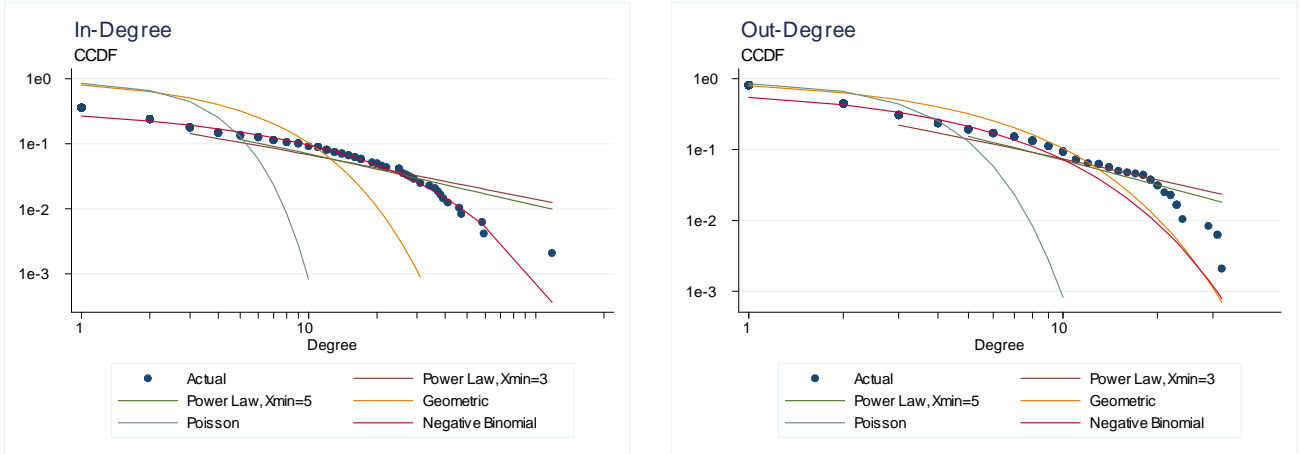

Figure 12: Complement of the CDF of out degree and in degree for September 29, 2006.

Figure 12 displays the distributions of in-degree and out-degree for a representative day in our sample period. Specifically the graphs have in(out) degree on the horizontal axis and the complement of the cumulative distribution function (CCDF) on the vertical axis, $F(x)=P(X \geq x)$. For each day, we fit the in-degree and out-degree to several different discrete distributions, which are all defined on the set of positive integers. The distributions are the power law, geometric, Poisson and negative binomial distributions. Since the Poisson distribution does such a poor job of describing the distribution of node in-degree or out-degree, we can conclude that the addition of new connections between banks in the federal funds market does not follow

\footnotetext{
${ }^{12}$ In this model one starts with $n$ nodes, connecting every pair of nodes with probability $p$, which creates a network that has on average $p n(n-1) / 2$ links.
} 

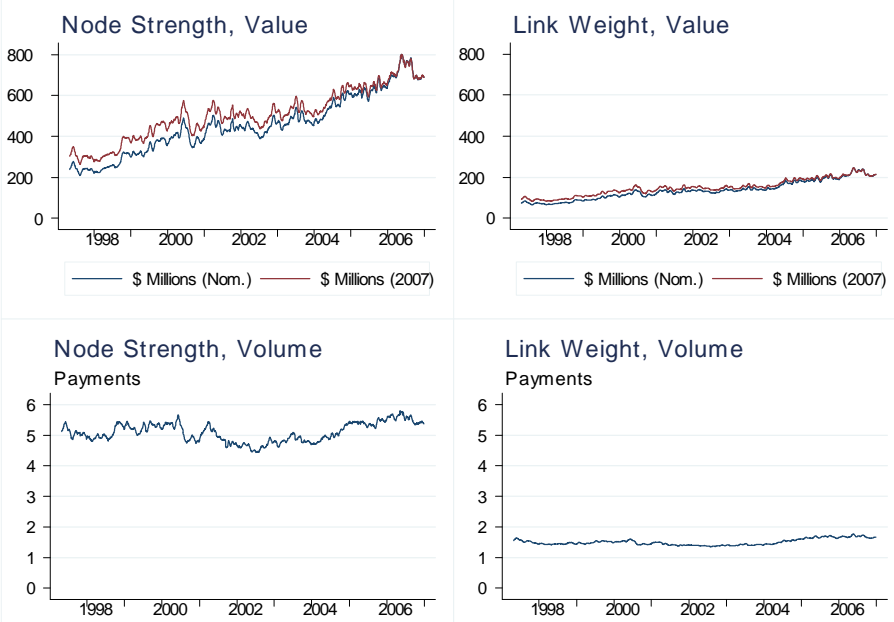

Figure 13: Average node strength and link weight, from April 1, 1997 to December 29, 2006. Top left: node strength, defined in terms of value. Top right: link weight, defined in terms of value. Bottom left: node strength, defined in terms of number of payments. Bottom right: link weight, defined in terms of number of payments.

the model of Erdos \& Renyi (1959). The power law distribution provides the best fit for out-degree, while the negative binomial distribution provides the best fit for in-degree. ${ }^{13}$

\subsection{Link weights and node strength}

Weights are attached to links in a network to differentiate the importance of each link. The strength of a node is the sum of the weights of all the links attached to it.

Definition: The in-strength of a node is the sum of the weights on incoming links from predecessors, $s_{i}^{i n}$ $=\sum_{j} w_{j i}$. The out-strength is the sum of the weights on outgoing links to successors, $s_{i}^{\text {out }}=\sum_{j} w_{i j}$. The average in-strength is equal to the average out-strength and equal to the sum of the link weights divided by the number of nodes, i.e., $\left\langle s^{\text {out }}\right\rangle=\frac{1}{n} \sum_{i} s_{i}^{\text {out }}=\frac{1}{n} \sum_{i} s_{i}^{i n}=\left\langle s^{i n}\right\rangle$. By definition, the ratio of average node strength to the average link weight is equal to the average degree of nodes in the network for a particular day.

Discussion: In 2006, the average weight per link was $\$ 219 \pm \$ 16$ million if weighted by value and $1.69 \pm 0.05$ payments if weighted by volume. In 2006 the average node strength was $\$ 719 \pm \$ 57$ million and $5.5 \pm 0.3$ payments, in terms of value and volume, respectively. So, the average bank sent 5.5 payments worth $\$ 720$ million each day. If a bank sent at least one loan to another bank on a particular day, then, on average, the former bank lent 1.69 payments worth $\$ 219$ million on that day. With respect to value, both node strength and link strength have been increasing steadily since 1997. Weighted by volume, node and link strength have been constant.

Banks in the core of the federal funds market have a higher node strength than other banks, even after we control for the number of counterparties per bank. We compute, for each day in 2006, the average and maximum strength in each component. Banks in the GSCC borrowed and lent greater than $\$ 5$ billion, on average, in 2006. Activity in the other components was much smaller. Even after we account for the difference in the number of counterparties between banks in the GSCC and other components, the nodes in the GSCC have a much higher strength than nodes in other components. For instance, while $\left\langle k_{i}^{i n}\right\rangle$, which

\footnotetext{
${ }^{13}$ For the daily distributions of in-degree the average estimated parameters for mean and variance were $\widehat{r}=0.154 \pm 0.023$ and $\widehat{p}=0.045 \pm 0.008$, respectively. The parameter estimate for the power law fit of the out-degree distribution was sensitive to the choice of $a$. When the whole out-degree distribution is fit to a power law the average parameter estimate was $\widehat{\gamma}=1.76 \pm 0.02$; if only nodes with out-degree greater than 2 are included the estimate was $\widehat{\gamma}=2.00 \pm 0.05$. Finally, if nodes with out-degree greater than four are fit to a power law distribution then the average parameter estimate was $\widehat{\gamma}=2.24 \pm 0.06$.
} 
is the average degree, is roughly 3.2 times larger in the GSCC relative to the GIN, $\left\langle s_{i}^{i n}\right\rangle$ is more than 9 times larger in the GSCC than in the GIN.

\begin{tabular}{crrrrr}
\hline & GSCC & GIN & GOUT & Tendril & DC \\
\hline$\left\langle s_{i}^{\text {in }}\right\rangle$ & 5,935 & 162 & 652 & 165 & 44 \\
$\left\langle s_{i}^{\text {out }}\right\rangle$ & 5,139 & 351 & 13 & 25 & 10 \\
$\max s_{i}^{\text {in }}$ & 65,630 & 914 & 9,011 & 1,180 & 70 \\
$\max s_{i}^{\text {out }}$ & 80,040 & 9,297 & 713 & 482 & 31 \\
\hline
\end{tabular}

Table 3: Average in-strength (measured in millions of dollars), average out-strength, maximum in-strength and maximum out-strength for each component. Each entry gives the average value across all networks in 2006.

The distributions of node strength have a heavy right tail (see Figure 14). Node strength, defined in terms of the number of payments a bank receives, follows a negative binomial distribution. If strength is defined in terms of the number of payments a bank sends, then the distribution of node strength follows a power law distribution. ${ }^{14}$ Finally, node strength, defined in terms of value sent or value received, follows a log-normal distribution (see Figure 14).
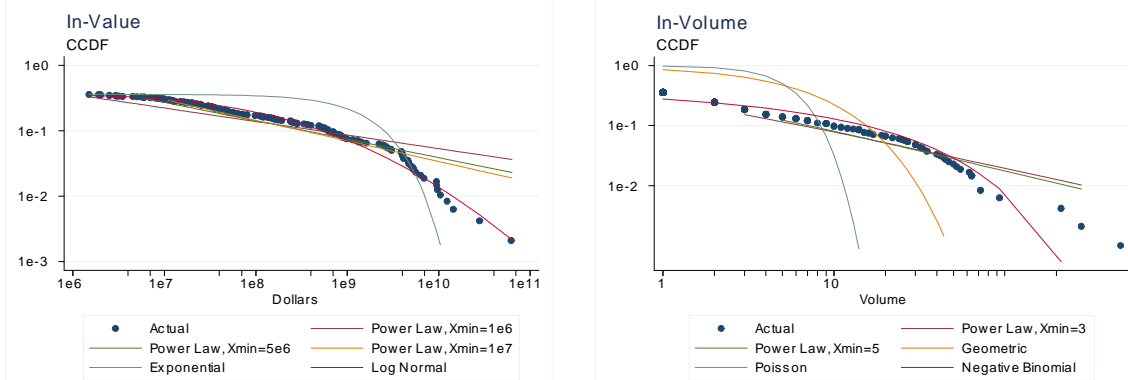

Figure 14: Complement of the CDF of node strength for Septmeber 29, 2006. In the left panel, node strength is set to the value received by the node. In the right panel, node strength is set to the number of payments received by the node. The distribution of strength, if defined by in-volume, follows a power law distribution while the distribution of nodes strength, if defined by in-value, follows a negative binomial (log-normal) distribution.

For each bank, for each day in 2006, we plot the bank's in(out)-degree against their in(out) node strength. Not surprisingly the two measures are highly correlated with one another. The log-log scaling of this figure obscures the fact that, for most degrees, the distribution of node strength conditional on the degree of the node has a very heavy right tail. The cloud of datapoints in the 200-400 payment, 30-60 in-degree range comes from one individual bank. Apparently, this bank borrows a larger than average number of loans from each counterparty. Likewise, a different bank lends a larger than average number of payments to each counterparty. In the lower two panels of Figure 15, we see an almost linear relationship between degree and strength. However, for banks that lend to more than thirty counterparties, the value and number of loans sent increases dramatically. Based on this figure, it seems reasonable (at least for small degree values) to regress the logarithm of node strength against the logarithm of node degree. For nodes with in-degree less than 30, a doubling node in-degree corresponds to payments received increasing by a factor of 2.16 and value received increasing by a factor of 3.54 . When out-degree is less than 20 , a doubling of node out-degree leads payments and value sent to increase by factors of 2.10 and 3.51 , respectively.

\footnotetext{
${ }^{14}$ Similar to the out-degree distribution, the power law parameter estimate depends on the cutoff point, $a$. If only nodes that send greater than two payments are considered, then $\widehat{\gamma}=1.89 \pm 0.04$. Increasing the cutoff point will increase the estimate of $\gamma$
} 

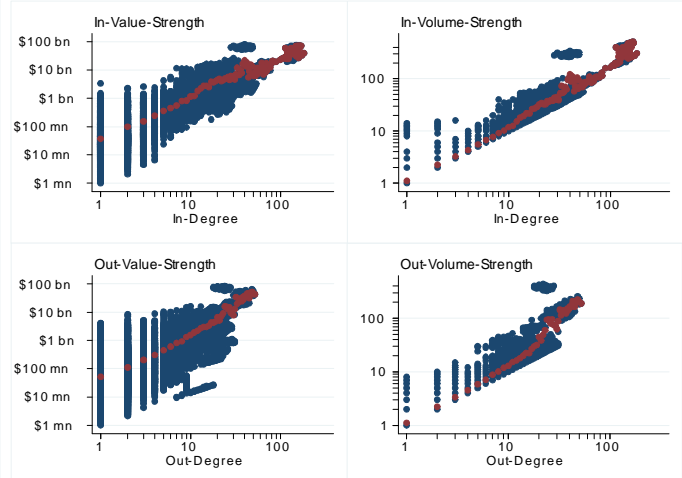

Figure 15: Relationship between node degree and node strength in 2006. Red dots are used to give the mean strength for each degree. Top left: in-value strength by in-degree. Top right: in-volume strength by in-degree. Bottom left: out-value strength by out-degree. Bottom right: out-volume strength by out-degree.

\subsection{Degree correlation}

Network analysis provides insights on trading patterns. In the federal funds market, the likelihood that a bank interacts with another bank depends on the characteristics of the two banks. Close competitors in a local market may choose not to trade with each other. As argued above, the notion of degree is a key characteristic of nodes. A network is said to be assortative if the degree of a node is positively related with the degrees of its neighbors, disassortative if the degree of a node is negatively related with the degrees of its neighbors, and uncorrelated if there is no relationship between the degree of a node and the degree of its neighbors.

Definition: Degree correlations refer to the notion that the probability that a node of degree $k_{i}$ is connected to another node of degree $k_{j}$ depends on the value of $k_{i}$. This correlation can be completely described by the joint degree distribution $\mathbb{P}\left(k_{i}, k_{j}\right)$. However, estimation can be difficult. In particular, any difficulties in terms of estimating the marginal degree distributions due to fat-tails are magnified when estimating the joint degree distribution. Hence, it is often more practical to compute the Pearson correlations between the degrees of the nodes at each end of a link (see Newman (2002)).

While the correlation between the degree of the nodes at each end of a link is one way to describe the assortivity of the federal funds network, a richer understanding comes when we compute the average degree of the neighbors of banks that have a particular degree. For example, the average in-degree of the successors of a node is

$$
\left\langle k_{\mathcal{S}}^{i n}\right\rangle_{i}=\frac{1}{k_{i}^{i n}} \sum_{j \in \mathcal{S}_{i}} k_{j}^{i n}
$$

and the average in-degree of the successors of nodes with out-degree $x$ is given by

$$
\left\langle k_{\mathcal{S}}^{\text {in }}\right\rangle(x)=\frac{1}{\sum_{i} 1\left(k_{i}^{\text {out }}=x\right)} \sum_{i}\left\langle k_{\mathcal{S}}^{\text {out }}\right\rangle_{i} 1\left(k_{i}^{\text {out }}=x\right)
$$

It is also possible to construct weighted versions of these measures. For example, the weighted average in-degree of the successors of a node is

$$
\left\langle\tilde{k}_{\mathcal{S}}^{i n}\right\rangle_{i}=\frac{1}{s_{i}^{i n}} \sum_{j \in \mathcal{S}_{i}} w_{i j} k_{j}^{i n}
$$

and the weighted average in-degree of the successors of nodes with out-degree $x$ is the average of $\left\langle\tilde{k}_{\mathcal{S}}^{\text {in }}\right\rangle_{i}$ across nodes in the network for which $k_{i}^{\text {out }}=x$. 

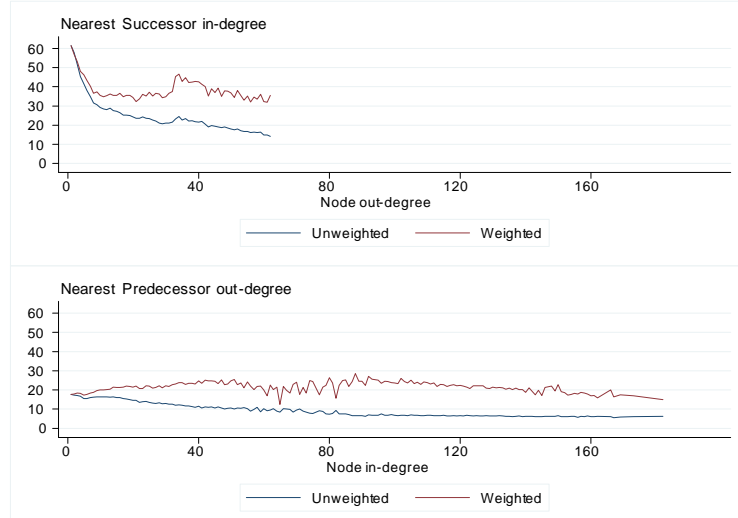

Figure 16: For 2006, the relationship between node in(out)-degree and the average out(in) degree of the neareset predecessor (successor).

Discussion: The federal funds network is disassortative. ${ }^{15}$ In 2006 , the Pearson correlation coefficients between the in- and out-degrees of two neighbors were all negative - ranging from -0.06 to -0.28 depending on the combination (see Table 5 on page 24). This implies that low degree banks are more likely to trade with high degree banks than other low degree banks. Weighting by the value lent across links decreases the disassortivity of the network. Since high degree banks are more likely to be associated with large-value links as shown in Figure 15, this suggests that even though small banks are not likely to interact with small banks, large banks do interact with large banks.

The correlation coefficients between node degrees at each end of a link do not give the full story. From the top panel of Figure 16, we see that the borrowers (i.e. the successors) from banks that lend to only a few other banks (i.e. low out-degree), are banks that borrow from many institutions (i.e. high in-degree). In other words, when a small bank is lending, the borrower is likely to be a larger bank that borrows from many institutions. Conversely, banks that lend to many other banks (i.e. high out-degree) are lending to institutions (i.e. its successors) who have relatively few counterparties that they borrow from. The blue line in the bottom panel tells a similar story. Banks that borrow from only a few counterparties (i.e. low in-degree) are borrowing from banks that lend to many institutions. On the other hand, if a bank is borrowing from a large number of institutions, it is borrowing from banks who are lending to a small number of counterparties. These relationships are most pronounced for degrees less than twenty. Finally, these relationships become weaker (or disappear) if we weight links by value.

\subsection{Clustering coefficient}

Another common measure of association between nodes is the probability that two nodes which are the neighbors of the same node, themselves share a link. This is equivalent to the observation that two people, each of whom is your friend, are likely to be friends with each other.

Definition: Following, Barrat et al. (2004) we define the weighted out-clustering coefficient of node $i$ as

$$
\widetilde{C}_{i}^{\text {out }}=\frac{1}{s_{i}^{\text {out }}\left(k_{i}^{\text {out }}-1\right)} \sum_{j, h} \frac{\left(w_{i j}+w_{i h}\right)}{2} a_{i j} a_{i h} a_{j h},
$$

where $w_{i j}$ is the weight on the link from node $i$ to node $j, k_{i}^{\text {out }}$ is the out-degree of node $i$ and $s_{i}^{\text {out }}$ is the out-strength of node $i$. The weighted in-clustering coefficient is defined analogously. For the unweighted clustering coefficient, $w_{i j}$ in Eq. 10 is replaced by $a_{i j}$ and $s_{i}$ is replaced by $k_{i}$. In a tree network, the clustering coefficient for each node is 0 , while in a classical random network the clustering coefficient is equal to the average degree divided by the number of nodes in the network. The clustering coefficients for the network

\footnotetext{
${ }^{15}$ Soramäki et al. (2006) find the same result for the Fedwire payments network.
} 


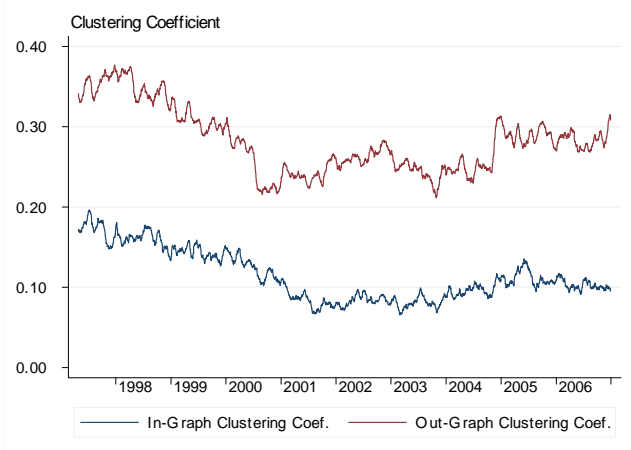

Figure 17: Network in/out clustering coeffiicient, from April 1, 1997 to December 29, 2006. The network clustering coefficient is the average of the clustering coefficient for the nodes in the network.

are obtained by taking the average across nodes. For example, the weighted out-clustering coefficient for a network is

$$
\widetilde{C}^{\text {out }}=\frac{1}{n} \sum_{i} \widetilde{C}_{i}^{\text {out }}
$$

Discussion: In 2006, the network in-clustering coefficient was $0.10 \pm 0.02$, and the out-clustering coefficient was $0.28 \pm 0.02$ (see Table 5). The two network clustering coefficients are highly correlated with one another over time. They are lower compared to their 1997 levels, and have been constant since 2001. The weighted versions of the network clustering coefficients are somewhat higher. The average weighted out-clustering coefficient was $0.30 \pm 0.02$ in 2006 . The weighted in-clustering coefficient was $0.13 \pm 0.02$. So, two of bank i's successors(predecessors) are slightly more likely to be connected if the amount lent (borrowed) by bank i to these two counterparties is relatively large.

In the GSCC the average in-clustering coefficient was 0.14. The average out-clustering coefficient for the GSCC was 0.29. To put this in the terminology of the federal funds market, if a bank in the GSCC lends to two institutions, the probability that these two institutions interact with each other is $29 \%$. The in-clustering coefficient in the GOUT is in line with that of the GSCC and the out-clustering coefficient of the GIN is the same as that of the GSCC. Banks in the GIN will be lending to banks in the GSCC or banks in the GIN. The out-clustering coefficient of a bank in the GIN is increased by the large number of interactions between the banks in the core of the federal funds market. Conversely, the in-clustering coefficient in the GIN is very low. This stems from the fact that lenders greatly outnumber borrowers within the GIN. If a bank in the GIN is borrowing from two other banks, these other banks must also be in the GIN. Since it is so unlikely for these two banks to be interacting with each other, the in-clustering coefficient of the first bank will be low. Finally, the clustering in the tendrils is significantly lower than in other components.

\begin{tabular}{lrrrrr}
\hline & GSCC & GIN & GOUT & Tendril & DC \\
\hline$C^{\text {in }}$ & 0.136 & 0.005 & 0.128 & 0.009 & 0 \\
$C^{\text {out }}$ & 0.289 & 0.298 & 0.012 & 0.022 & 0 \\
$\widetilde{C}^{\text {in }}$ & 0.196 & 0.005 & 0.146 & 0.009 & 0 \\
$\widetilde{C}^{\text {out }}$ & 0.345 & 0.308 & 0.012 & 0.022 & 0 \\
\hline
\end{tabular}

Table 4: Average in-degree, average out-degree, maximum in-degree and maximum out-degree for each component. Each figure is the average across all networks in 2006.

\section{7 $\quad$ Distance and diameter}

Many real world networks exhibit what is called the small world property, where most nodes can be reached from the others via a small number of links. The small world property extant in the federal funds network tells us that the degree of intermediation between net demanders of federal funds and net suppliers is small. 
Definition: The distance from node $i$ to node $j, d_{i j}$, is defined to be the length of the shortest directed path from node $i$ to node $j$. If there is no path from node $i$ to node $j, d_{i j}$ is set to $\infty$. The average in-path length of node $i,\left\langle l_{i}^{i n}\right\rangle$, is equal to the mean of $\left\{d_{j i}: d_{j i}<\infty\right\}$. Likewise, the average out-path length, of node $i,\left\langle l_{i}^{\text {out }}\right\rangle$, is equal to the mean of $\left\{d_{i j}: d_{i j}<\infty\right\}$. The eccentricity of a node is the maximum distance from that node to any other node in the network. The in-eccentricity is $\varepsilon_{i}^{i n}=\max \left(\left\{d_{j i}: d_{j i}<\infty\right\}\right)$ and out-eccentricity is $\varepsilon_{i}^{\text {out }}=\max \left(\left\{d_{i j}: d_{i j}<\infty\right\}\right)$. The diameter of a network is equal to the maximum eccentricity over all nodes in the network: $D=\max \left(\varepsilon_{i}^{\text {in }}\right)=\max \left(\varepsilon_{i}^{\text {out }}\right) .{ }^{16}$

Discussion: In 2006 the average in-path length was equal to $2.4 \pm 0.2$, while the average out-path length was equal to $2.7 \pm 0.1$. The average in-eccentricity was equal to $4.1 \pm 0.5$ and the average out-eccentricity was equal to $4.5 \pm 0.6$. This means that, for the average bank in the federal funds market, banks that lend federal funds can expect to have those funds lent out up to $3.5(=4.5-1)$ more times. Also, for the average borrower in the federal funds market, the funds that the bank borrows had switched hands up to 3.1 more times. Finally, the network diameter averaged $7.3 \pm 1.0$ in 2006. These numbers have been relatively constant over the sample period. Average out-path length and out-eccentricity have been consistently greater than the average in-path length and in-eccentricity. This relates to the asymmetric structure of the federal funds market. Previous studies have described the long chain of transactions where small regional banks lend to medium sized banks who then lend to large money-center banks. These large banks will lend to each other or, possibly, lend to medium sized banks (see Furfine (1999) or Stigum (1990)). While it may take a long time for funds to accumulate and reach the money-center banks, once they arrive they are quickly dispersed to those who demand them.

\footnotetext{
${ }^{16}$ The average in- and out-path length for the network are $\left\langle l^{\text {in }}\right\rangle=\frac{1}{n} \sum_{i}\left\langle l_{i}^{\text {in }}\right\rangle$ and $\left\langle l^{\text {out }}\right\rangle=\frac{1}{n} \sum_{i}\left\langle l_{i}^{\text {out }}\right\rangle$, respectively. The average in- and out-eccentricities for the network are $\left\langle\varepsilon^{i n}\right\rangle=\frac{1}{n} \sum_{i} \varepsilon_{i}^{\text {in }}$ and $\left\langle\varepsilon^{\text {out }}\right\rangle=\frac{1}{n} \sum_{i} \varepsilon_{i}^{\text {out }}$, respectively.
} 


\begin{tabular}{|c|c|c|c|c|c|c|c|c|c|c|c|}
\hline & Mean & Med & Min & $\operatorname{Max}$ & $\mathrm{SD}$ & & Mean & Med & Min & Max & SD \\
\hline \multicolumn{6}{|l|}{ Payments } & \multicolumn{6}{|l|}{ Distance Measures } \\
\hline Volume $(, 000)$ & 2.6 & 2.6 & 1.0 & 3.0 & 0.1 & $\left\langle l^{i n}\right\rangle$ & 2.4 & 2.4 & 2.0 & 3.0 & 0.2 \\
\hline Value (\$bn) & 338.1 & 336.9 & 140.1 & 421.3 & 25.7 & $\left\langle l^{\text {out }}\right\rangle$ & 2.7 & 2.7 & 2.3 & 3.2 & 0.1 \\
\hline Average (\$mn) & 129.7 & 128.9 & 114.0 & 150.0 & 7.0 & $\left\langle\varepsilon^{i n}\right\rangle^{\prime}$ & 4.1 & 4.1 & 3.1 & 6.1 & 0.5 \\
\hline Median (\$mn) & 50.2 & 50.0 & 50.0 & 60.0 & 1.2 & $\left\langle\varepsilon^{\text {out }}\right\rangle$ & 4.5 & 4.4 & 3.6 & 6.6 & 0.6 \\
\hline \multicolumn{6}{|l|}{ Components } & $D$ & 7.3 & 7 & 5 & 10 & 1.0 \\
\hline GWCC & 463.0 & 463 & 302 & 500 & 15.3 & \multicolumn{6}{|l|}{ Degree Distribution } \\
\hline $\mathrm{DC}$ & 7.1 & 8 & 0 & 16 & 3.8 & $\left\langle k_{i}^{\text {out }}\right\rangle=\left\langle k_{i}\right\rangle$ & 3.3 & 3.3 & 2.1 & 3.5 & 0.1 \\
\hline GSCC & 47.2 & 47 & 28 & 66 & 5.7 & $\operatorname{Max} k_{i}^{\text {out }}$ & 48.8 & 49.0 & 32.0 & 62.0 & 6.4 \\
\hline GIN & 270.1 & 269 & 123 & 340 & 27.5 & $\operatorname{Max} k_{i}^{i n}$ & 127.6 & 125.5 & 64.0 & 182.0 & 16.3 \\
\hline GOUT & 79.0 & 78 & 36 & 119 & 16.0 & $\hat{\gamma}(\mathrm{a}=2)$ & 2.00 & 2.05 & 1.90 & 2.44 & 0.05 \\
\hline Tendrils & 66.8 & 66 & 34 & 103 & 12.9 & $\hat{r}$ & 0.15 & 0.11 & 0.10 & 0.24 & 0.02 \\
\hline \multicolumn{6}{|c|}{ Nodes, Links, Deg. of Completeness and Reciprocity } & $\hat{p}$ & 0.05 & 0.03 & 0.03 & 0.11 & 0.01 \\
\hline Links & 1543 & 1543 & 634 & 1696 & 72.2 & \multicolumn{6}{|l|}{ Degree Correlation } \\
\hline Nodes & 470.2 & 470 & 307 & 511 & 15.3 & $\operatorname{Cor}\left(k_{i}^{\text {out }}, k_{j}^{i n}\right)$ & -0.28 & -0.28 & -0.33 & -0.20 & 0.02 \\
\hline$\alpha(\%)$ & 0.70 & 0.70 & 0.62 & 0.80 & 0.03 & W.Cor $\left(k_{i}^{\text {out }}, k_{j}^{\text {in }}\right)$ & -0.06 & -0.06 & -0.17 & 0.07 & 0.04 \\
\hline$\varrho(\%)$ & 6.5 & 6.5 & 4.6 & 8.5 & 0.8 & $\operatorname{Cor}\left(k_{i}^{i n}, k_{j}^{i n}\right)$ & -0.13 & -0.13 & -0.16 & -0.10 & 0.01 \\
\hline$\widetilde{\varrho}(\%)$ & 43.0 & 43.4 & 30.2 & 48.9 & 3.2 & $\mathrm{~W} . \operatorname{Cor}\left(k_{i}^{i n}, k_{j}^{i n}\right)$ & -0.12 & -0.12 & -0.22 & -0.01 & 0.04 \\
\hline$\rho(\%)$ & 5.8 & 5.8 & 3.9 & 7.8 & 0.8 & \multicolumn{6}{|c|}{ Link Weights and Node Strength } \\
\hline$\widetilde{\rho}(\%)$ & 7.0 & 6.9 & 2.9 & 12.4 & 1.7 & $\langle w\rangle(\$ \mathrm{mn})$ & 219 & 217 & 186 & 274 & 16.3 \\
\hline \multicolumn{6}{|l|}{ Clustering } & $\langle w\rangle$ (Volume) & 1.69 & 1.68 & 1.59 & 1.85 & 0.05 \\
\hline$C^{i n}$ & 0.10 & 0.10 & 0.05 & 0.14 & 0.02 & $\langle s\rangle(\$ \mathrm{mn})$ & 719 & 713 & 456 & 900 & 56.8 \\
\hline$\widetilde{C}^{i n}$ & 0.13 & 0.13 & 0.07 & 0.17 & 0.02 & $\langle s\rangle$ (Volume) & 5.54 & 5.53 & 3.40 & 6.23 & 0.26 \\
\hline$C^{\text {out }}$ & 0.28 & 0.28 & 0.19 & 0.36 & 0.02 & $\hat{\gamma}(a=2)$ & 1.89 & 1.95 & 1.80 & 2.16 & 0.04 \\
\hline \multirow[t]{2}{*}{$\widetilde{C}^{\text {out }}$} & 0.30 & 0.30 & 0.21 & 0.38 & 0.02 & $\hat{r}$ & 0.12 & 0.10 & 0.09 & 0.17 & 0.02 \\
\hline & & & & & & $\hat{p}$ & 0.02 & 0.02 & 0.01 & 0.05 & 0.00 \\
\hline
\end{tabular}

Table 5: Summary statistics for network characteristics, 2006. Components: GWCC = giant weakly connected component, GSCC = giant strongly connected component, DC = disconnected components, GIN = giant in-component, GOUT = giant-out component. Nodes, Links, Degree of Completeness and Reciprocity: $\alpha=$ Degree of Completeness, $\varrho=$ Reciprocity, $\rho=$ Correlation coefficient of adjacency matrix. Distance Measures: $\langle l\rangle=$ average path-length, $\langle\epsilon\rangle=$ average eccentricity, $D=$ diameter. Degree Distribution: $k$ $=$ degree, $\hat{\gamma}=$ Power Law estimate for out-degree distribution, $\hat{r}, \hat{p}=$ Parameter estimates for fitting indegree to negative binomial distribution. Degree Correlation (4 of 8 possible combinations given): $k_{i}^{\text {out }}=$ Out-degree of sending institution, $k_{i}^{i n}=$ In-degree sending instituion, $k_{j}^{i n}=$ In-degree of receiving institution. Link Weights and Node Strength: $w=$ link weights, $s=$ node strength, $\hat{\gamma}=$ Estimate of power law coefficient for distribution of node strength, defined by number of payments sent. $\hat{r}, \hat{p}=$ Estimate of negative binomial parameters for distribution of node strength, defined by number of payments received.

\subsection{Node centrality}

Within network analysis, centrality measures are used to determine the relative importance of a node within the network. The Bonacich centrality of a node has been shown to predict bargaining power in exchange networks (see Borgatti \& Everett (2005)). Here we explore whether such measures can help predict the rates that banks are paying to borrow or being paid to lend to one another.

Definition: Node centrality measures are used to identify the position of each bank in the federal funds market. We use a centrality measure that was introduced in Bonacich (1987). The Bonacich centrality of node $i$ is equal to the $i^{\text {th }}$ entry of

$$
b(\alpha, \beta, \boldsymbol{W})=\alpha[\boldsymbol{I}-\beta \boldsymbol{W}]^{-1} \mathbf{W} \mathbf{1}
$$

where $\alpha$ is a scaling parameter used to set the length of $b$ equal to the number of nodes in the network (see Bonacich (1987)), $\boldsymbol{I}$ is the identity matrix, $\beta$ is a decay factor, $\boldsymbol{W}$ is the weight matrix defined in section 5 and 1 is a vector of ones. The parameter $\beta$ can take values in the range $\left[-\frac{1}{|L|}, \frac{1}{|L|}\right]$, where $L$ is the eigenvalue of $\boldsymbol{W}$ which has the largest absolute value. This centrality measure incorporates the lending activity of a bank as well as the lending activity of its successors. Banks that lend large sums of money will, for the most 
part, have large centrality scores. Depending on the parameter, $\beta$, the centrality scores of a bank's neighbor can positively or negatively affect the bank's centrality score. For $\beta=0$ the Bonacich centrality of a node is independent of the Bonacich centralities of their neighbors: the Bonacich centrality is proportional to the out-strength of the node. For $\beta>0$ central nodes have a larger positive effect on the Bonacich centrality of their neighbors than nodes with low centrality scores do. Banks that lend a lot of money and/or banks that lend to counterparties that have high lending activity will have the highest Bonacich centrality scores. As $\beta$ gets bigger, the effect of a node's centrality on its neighbors becomes more pronounced. Conversely, for $\beta<0$, a node will have a high Bonacich centrality score if it shares links with many nodes with low centrality scores.

Using $\boldsymbol{W}^{T}$ instead of $\boldsymbol{W}$ in the calculations will give centrality scores with a different, but equally valid, interpretation. When $\boldsymbol{W}$ is used, banks that lend a large amount of reserves will have a high centrality score. However, it is equally valid to believe that banks that are the biggest players on the borrowing side of the market should be defined as the most central. We use $b_{i}\left(\alpha, \beta, \mathbf{W}^{T}\right)$ to denote the Bonacich centrality of a node, where $\boldsymbol{W}^{T}$ is used as the weight matrix. Using $\boldsymbol{W}^{T}$ is equivalent to using $\boldsymbol{W}$, with the direction of all of the links reversed.

Example: In this example we use activity from a half-hour interval to show how these centrality scores are computed. Figure 18 presents the network of federal funds activity that took place between 8:30 and 9:00 a.m. on January 31, 2006 both as a graph and as a weight matrix. High-value links are marked in yellow, while low-value links are colored red. Between 8:30 and 9:00 the largest payments are made by node 3 (to node 6 ) and node 5 (to node 3 ). All nodes receive at least one payment, but three of the nodes (1, 4 and 7 ) do not initiate any payments.

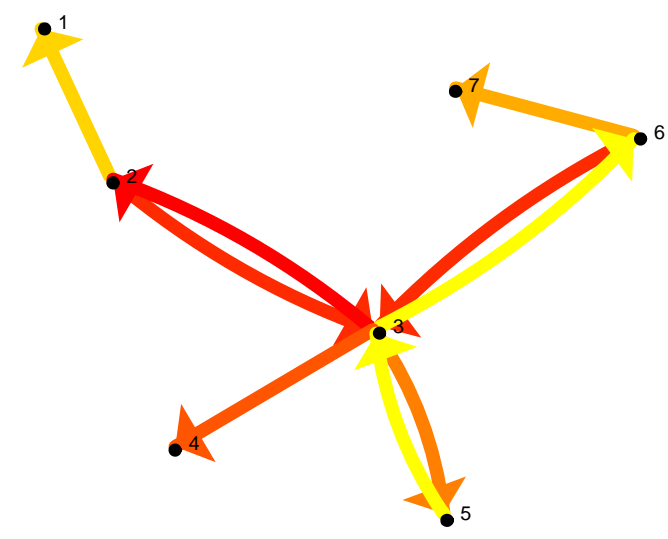

\begin{tabular}{|c|ccccccc|}
\hline$i j$ & 1 & 2 & 3 & 4 & 5 & 6 & 7 \\
\hline 1 & 0 & 0 & 0 & 0 & 0 & 0 & 0 \\
2 & 450 & 0 & 10 & 0 & 0 & 0 & 0 \\
3 & 0 & 1 & 0 & 25 & 35 & 750 & 0 \\
4 & 0 & 0 & 0 & 0 & 0 & 0 & 0 \\
5 & 0 & 0 & 750 & 0 & 0 & 0 & 0 \\
6 & 0 & 0 & 10 & 0 & 0 & 0 & 200 \\
7 & 0 & 0 & 0 & 0 & 0 & 0 & 0 \\
\hline
\end{tabular}

Figure 18: Left: Federal funds network on January 31, 2006, between 8:30 and 9:00 a.m. Yellow denotes high-value links. Red is used for low-value links. Right: The matrix, W, for the same period of time.

The Bonacich centrality scores for different values of $\beta$ are computed in Table 6 . We will allow $\beta$ $\in\left\{-\frac{1}{L},-\frac{1}{2 L}, 0, \frac{1}{2 L}, \frac{1}{L}\right\}$. Nodes 1,4 and 7 have Bonacich centrality scores of 0 when $\boldsymbol{W}$ is used in the calculation of node centrality. For four of the specifications, node 3 is the most central node. When $\beta$ is positive $b\left(\alpha, \beta, \mathbf{W}^{T}\right)$ is greater for 6 and 7 than it is for node 3 . The high value link from node 3 to node 6 is the cause. When $\beta$ is negative, nodes that share high value links with node 3 are penalized. For example, node 6 receives a large value payment to node 3 , the most central node in terms of $b(\alpha, \beta, \mathbf{W})$. The centrality of node 6 falls as $\beta$ decreases. This, in turn, causes the centrality of node 7 to increase as $\beta$ becomes more negative. 


\begin{tabular}{|c|rrrrr|rrrrr|}
\hline & \multicolumn{9}{|c|}{$b(\alpha, \beta, \mathbf{W})$} \\
$i$ & $1 / L$ & $1 / 2 L$ & 0 & $-1 / 2 L$ & $-1 / L$ & $1 / L$ & $1 / 2 L$ & 0 & $-1 / 2 L$ & $-1 / L$ \\
\hline 1 & 0.02 & 0.83 & 2.66 & 1.87 & 0.02 & 0.00 & 0.00 & 0.00 & 0.00 & 0.00 \\
2 & 0.01 & 0.01 & 0.01 & -0.01 & -0.01 & 0.09 & 0.76 & 2.65 & 4.84 & 0.09 \\
3 & 1.14 & 2.10 & 4.56 & 3.74 & 1.14 & 1.67 & 2.60 & 4.67 & 4.46 & -1.67 \\
4 & 0.16 & 0.19 & 0.15 & -0.15 & -0.16 & 0.00 & 0.00 & 0.00 & 0.00 & 0.00 \\
5 & 0.22 & 0.26 & 0.21 & -0.21 & -0.22 & 6.80 & 6.44 & 4.32 & -1.00 & 6.80 \\
6 & 4.67 & 5.65 & 4.44 & -4.53 & -4.67 & 0.09 & 0.38 & 1.21 & 2.15 & 0.09 \\
7 & 5.08 & 3.44 & 1.18 & 3.30 & 5.08 & 0.00 & 0.00 & 0.00 & 0.00 & 0.00 \\
\hline
\end{tabular}

Table 6: Bonacich centrality scores for the network of Figure 18, using different decay parameters and weight matrices.

Discussion: We regress the rate of a federal funds loan on the difference in centrality scores of the borrower and lender. We suspect that, if the borrowing bank has a higher centrality score than the lending bank, it will be able to extract a lower interest rate from its counterparty. Conversely, if the lending institution has the higher centrality score, the interest rate on the loan will be higher. We run the following regressions for each centrality measure:

$$
\begin{aligned}
r= & \left.\gamma_{0}+\gamma_{1} 1 \text { (Centrality of lender }>\text { Centrality of borrower }\right)+ \\
& \left.\gamma_{2} 1 \text { (Assets of lender }>\text { Assets of borrower }\right)+\gamma_{3} \text { Loan Size }+ \\
& +\gamma_{4} \text { Time of Settlement }+\gamma_{5} \text { Duration of Loan }+\varepsilon
\end{aligned}
$$

where $r$ is interest rate on the loan minus the effective federal funds rate on that day. The centrality and assets measures are computed based on data from the previous month. The results from the regressions are presented in Table 7 . The adjusted $R^{2}$ values vary from $2.2 \%$ to $3.7 \%$. All coefficients have the expected signs with the exception of the time of settlement where the sign depends on the specification. ${ }^{17} \mathrm{~A}$ loan with longer duration will, on average, have a higher interest rate. The interest rate on a given loan is approximately $0.25-0.4$ basis points higher for every additional hour that the loan is held. Larger loans imply a higher interest rate. Loans where the lender has a larger asset size than the borrower have a higher interest rate by about 2 basis points. Banks with higher centrality scores command better rates, even after controlling for size, in terms of assets. All centrality measures are statistically significant-with a p-value less that .001- in determining the rate charged between the two banks. The results are also economically significant. Loans where the lender has a higher centrality score have an interest rate that is roughly 2 to 4 basis points higher. For a $\$ 50$ million overnight loan an extra 3 basis points is equivalent to an additional $\$ 42$ in interest. In other words, if a bank is important on the lending side of the market then it can command good rates. The preferred specifications, in terms of explaining the variation in interest rates across loans (i.e. maximizing $R^{2}$ ), are the regressions that have $\beta$ in the range $-1 / 2 L$ and $1 / 2 L$ and use $\boldsymbol{W}$ in computing the Bonacich centrality. For the sake of comparison, we also ran regressions using in-degree, out-degree or strength - in terms of in-volume or out-volume - as our "centrality measures". All of these specifications were dominated by those using Bonacich measures with $\boldsymbol{W}$ and $\beta$ within the range listed above.

\footnotetext{
${ }^{17}$ The constant term is negative and highly signigicant in all regressions. The results of the regressions are nearly identical whether or not calendar effects are included.
} 


\begin{tabular}{|c|c|c|c|c|c|c|c|}
\hline \# & Centrality Measure (CM) & 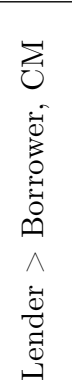 & 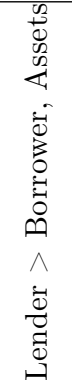 & 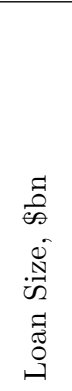 & 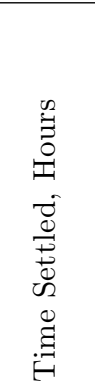 & 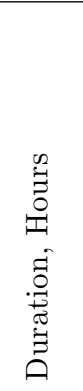 & 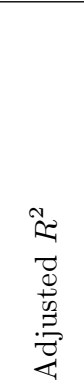 \\
\hline 1 & $b_{i}\left(\alpha, 1 / L, \mathbf{W}^{T}\right)$ & 1.86 & 1.81 & 1.22 & 0.02 & 0.38 & 0.025 \\
\hline 2 & $b_{i}\left(\alpha, 1 / 2 L, \mathbf{W}^{T}\right)$ & 1.89 & 1.80 & 1.23 & 0.02 & 0.38 & 0.025 \\
\hline 3 & $b_{i}\left(\alpha, 0, \mathbf{W}^{T}\right)$ & 1.86 & 1.79 & 1.21 & 0.02 & 0.38 & 0.025 \\
\hline 4 & $b_{i}\left(\alpha,-1 / 2 L, \mathbf{W}^{T}\right)$ & 1.47 & 1.87 & 1.13 & 0.02 & 0.38 & 0.023 \\
\hline 5 & $b_{i}\left(\alpha,-1 / L, \mathbf{W}^{T}\right)$ & 1.13 & 1.99 & 1.13 & 0.02 & 0.38 & 0.022 \\
\hline 6 & $b_{i}(\alpha, 1 / L, \mathbf{W})$ & 3.10 & 1.86 & 0.79 & -0.07 & 0.25 & 0.034 \\
\hline 7 & $b_{i}(\alpha, 1 / 2 L, \mathbf{W})$ & 3.37 & 1.86 & 0.79 & -0.07 & 0.24 & 0.036 \\
\hline 8 & $b_{i}(\alpha, 0, \mathbf{W})$ & 3.42 & 1.85 & 0.80 & -0.08 & 0.24 & 0.037 \\
\hline 9 & $b_{i}(\alpha,-1 / 2 L, \mathbf{W})$ & 2.52 & 1.83 & 1.04 & -0.02 & 0.30 & 0.036 \\
\hline 10 & $b_{i}(\alpha,-1 / L, \mathbf{W})$ & 1.76 & 2.21 & 1.27 & -0.03 & 0.36 & 0.026 \\
\hline 11 & $s_{i}^{i n}$, volume & 1.50 & 1.87 & 1.09 & 0.02 & 0.38 & 0.023 \\
\hline 12 & $s_{i}^{\text {out }}$, volume & 2.97 & 2.03 & 1.09 & -0.06 & 0.27 & 0.033 \\
\hline 13 & $k_{i}^{i n}$ & 1.29 & 1.96 & 1.13 & 0.38 & 0.38 & 0.023 \\
\hline 14 & $k_{i}^{\text {out }}$ & 1.55 & 2.29 & 1.36 & 0.36 & 0.36 & 0.025 \\
\hline
\end{tabular}

Table 7: Coefficient estimates for the regression of equation 13. All coefficients except for time settled are significant with a p-value less that 0.001. The coefficient estimate for time settled has a p-value less than 0.001 for regressions 6 to 10, 12, and 14 and a p-value less than 0.05 for regressions 2, 3, 4, 11 , and 13 .

\section{Conclusion}

In this paper, we analyze the topology of the 2,415 daily networks formed by overnight federal funds loans between commercial banks and other financial institutions over the period April 1, 1997 to December 29, 2006. These networks share many of the characteristics found in other complex networks. We identify two relationships between network statistics and factors external to the network. First, we find that reciprocity is correlated with the federal funds rate. Second, Bonacich centrality is useful in explaining some of the price heterogeneity displayed in the federal funds market.

Much of the literature on complex networks focuses on the vulnerability of networks to disruptions and the mechanisms of contagion. This is analogous to the financial literature on systemic risk. However, little is known about the empirical properties of networks used to model financial markets under stress. We leave an investigation of the properties of the federal funds network under times of turmoil (such as 9/11 or August 2007) for future research.

\section{References}

Albert, Reka, \& Barabási, Albert-László. 2002. Statistical Mechanics of Complex Networks. Reviews of Modern Physics, 74, 48-94.

Albert, Reka, Jeong, Hawoong, \& Barabási, Albert-László. 2000. Error and Attack Tolerance of Complex Networks. Nature, 406, 378-382.

Allen, Franklin, \& Gale, Douglas. 2000. Financial contagion. Journal of Political Economy, 108(1), 1-33.

Allen, Linda, \& Saunders, Anthony. 1986. The large-small bank dichotomy in the federal funds market. Journal of Banking \& Finance, 10(2), 219-230. 
Allen, Linda, Peristiani, Stavros, \& Saunders, Anthony. 1989. Bank Size, Collateral, and Net Purchase Behavior in the Federal Funds Market: Empirical Evidence. Journal of Business, 62(4), 501-515.

Barrat, Alain, Barthelemy, Marc, Pastor-Satorras, Romualdo, \& Vespignani, Alessandro. 2004. The Architecture of Complex Weighted Networks. Proceedings of the National Academy of Sciences of the United States of America (PNAS), 101, 3747.

Bartolini, Leonardo, Gudell, Svenja, Hilton, Spence, \& Schwarz, Krista. 2005a. Intra-Day Behavior of the Federal Funds Market. Federal Reserve Bank of New York Staff Reports.

Bartolini, Leonardo, Gudell, Svenja, Hilton, Spence, \& Schwarz, Krista. 2005b. Intraday Trading in the Overnight Federal Funds Market. Federal Reserve Bank of New York Current Issues in Economics and Finance, 11(11), 1-7.

Bartolini, Leonardo, Hilton, Spence, \& McAndrews, James. 2008. Settlement delays in the money market. Federal Reserve Bank of New York Staff Reports.

Bonacich, Phillip. 1987. Power and Centrality: A Family of Measures. The American Journal of Sociology, 92(5), 1170-1182.

Borgatti, Stephen P., \& Everett, Martin G. 2005. A Graph-theoretic perspective on Centrality. Social Networks, 28(4), 466-484.

Boss, Michael, Elsinger, Helmut, Summer, Martin, \& Thurner, Stefan. 2004. The Network Topology of the Interbank Market. Computer Networks and ISDN Systems, 4, 677-684.

Broder, Andrei, Kumar, Ravi, Maghoul, Farzin, Rajagopalan, Sridhar, Stata, Raymie, Tomkins, Andrew, \& Wiener, Janet. 2000. Graph Structure in the Web.

Costa, Luciano da F., Rodrigues, Francisco A., Travieso, Gonzalo, \& Boas, P. R. Villas. 2006. Characterization of complex networks: A survey of measurements. arXiv:cond-mat/0505185.

Demiralp, Selva, Preslopsky, Brian, \& Whitesell, William C. 2004. Overnight Interbank Loan Markets. Finance and Economics Discussion Series: Federal Reserve Board of Governors.

Dorogovtsev, S.N., Mendes, J.F.F., \& Samukhin, A.N. 2001. Giant Strongly Connected Component of Directed Networks. Physical Review E, 64, 025101.

Erdos, Paul, \& Renyi, Alfred. 1959. On Random Graphs. Publicationes Mathematicae, 6, 290-297.

Furfine, Craig H. 1999. The Microstructure of the Federal Funds Market. Financial Markets, Institutions, and Instruments, 8(5), 24-44.

Gale, Douglas M, \& Kariv, Shachar. 2007. Financial Networks. The American Economic Review, 97(2), 99-103.

Hamilton, James D. 1996. The Daily Market for Federal Funds. Journal of Political Economy, 104(1), 26-56.

Ho, Thomas S. Y., \& Saunders, Anthony. 1985. A Micro Model of the Federal Funds Market. Journal of Finance, 40(3, Papers and Proceedings of the Forty-Third Annual Meeting American Finance Association, Dallas, Texas, December 28-30, 1984), 977-988.

Inaoka, Hajime, Ninomiya, Takuto, Taniguchi, Ken, Shimizu, Tokiko, \& Takayasu, Hideki. 2004. Fractal Network Derived from Banking Transaction: An Analysis of Network Structures Formed by Financial Institutions. Bank of Japan Working Papers.

Iori, Giulia, de Masi, Giulia, Precup, Ovidiu V., Gabbi, Giampaolo, \& Caldarelli, Guido. 2005 (January 20, 2005). The Microstructure of the Italian Overnight Money Market. Department of Economics Discussion Paper Series 05/05. City University London. 
Meulendyke, Ann-Marie. 1998. U.S. Monetary Policy and Financial Markets. Federal Reserve Bank of New York.

Newman, M E J. 2002. Assortative mixing in networks. Physical Review Letters, 89, 208701.

Newman, M.E.J. 2003. The Structure and Function of Complex Networks. SIAM Review, 45(2), 167-255.

Portes, Richard, \& Rey, Helene. 2005. The determinants of cross-border equity flows. Journal of International Economics, 65(2), 269-296.

Rosati, Simonetta, \& Secola, Stefania. 2005. Explaining cross-border large-value payment flows - evidence from TARGET and EURO 1 data. ECB Working Paper Series.

Soramäki, Kimmo, Bech, Morten L., Arnold, Jeffrey, Glass, Robert J., \& Beyeler, Walter E. 2006. The Topology of Interbank Payment Flows. Physica A, 379(1), 317-333.

Stigum, Marcia. 1990. The Money Market. 3rd edn. Homewood, Ill.: Dow Jones-Irwin.

\section{A Calendar Effects}

Among other graph characteristics, Iori et al. (2005) look at the average degree and maximum degree of banks in the Italian overnight market with respect to the day of the maintenance period. They find that as one gets closer to the end of the maintenance period volume, bank participation, and number of links increase. We regress network statistics on the calendar effects used in Demiralp et al. (2004). The target federal funds rate and a trend term are also included as predictors. In the last two days of the maintenance period degree of completeness, reciprocity, value, average degree and the number of links are all significantly higher. The trend and target interest rate are always highly significant. For this reason, when stating point estimates of network statistics, we have focused only on 2006, where these two variables are not likely to have such a large effect.

\begin{tabular}{|c|c|c|c|c|c|c|c|}
\hline & 疍 & 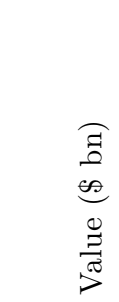 & 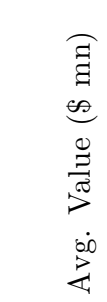 & $\underbrace{\widehat{d e}}_{0}$ & 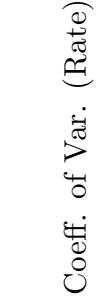 & $\tilde{\tilde{\Xi}}$ & \\
\hline Maint Day $=1$ & 1.435 & -2.161 & -0.539 & 0.000 & $\begin{array}{c}-0.001 \\
\end{array}$ & -0.005 & -0.004 \\
\hline Maint Day $=2$ & 20.39 & -4.568 & -1.978 & 0.003 & 0.005 & 0.000 & 0.004 \\
\hline Maint Day $=3$ & -0.922 & -0.471 & 0.174 & -0.001 & 0.000 & -0.002 & -0.003 \\
\hline Maint Day $=4$ & 8.910 & 2.585 & 0.285 & 0.002 & 0.000 & 0.001 & -0.002 \\
\hline Maint Day $=6$ & 2.893 & -3.513 & -0.985 & 0.000 & 0.001 & -0.003 & -0.002 \\
\hline Maint Day $=7$ & 38.607 & -1.800 & -1.958 & 0.003 & 0.007 & -0.001 & 0.006 \\
\hline Maint Day $=8$ & 7.306 & 0.006 & -0.108 & -0.001 & 0.002 & -0.001 & 0.001 \\
\hline Maint Day $=9$ & 18.650 & 4.824 & 0.370 & 0.004 & 0.009 & 0.001 & 0.000 \\
\hline Maint Day $=10$ & 33.650 & 5.749 & 0.125 & 0.012 & 0.017 & 0.001 & 0.005 \\
\hline After 1 Day Holiday & -6.340 & -1.641 & 0.631 & 0.001 & 0.006 & 0.003 & 0.005 \\
\hline After 3 Day Holiday & 22.378 & 3.085 & -0.142 & -0.004 & -0.001 & 0.000 & 0.003 \\
\hline Before 1 Day Holiday & 6.860 & -8.488 & -1.300 & 0.004 & 0.005 & -0.009 & 0.002 \\
\hline Before 3 Day Holiday & 34.252 & 3.282 & -0.904 & 0.003 & 0.008 & 0.006 & 0.008 \\
\hline 1st of Month & 65.456 & 10.182 & -0.536 & -0.001 & 0.009 & -0.001 & 0.011 \\
\hline 15th of Month & 42.347 & 5.881 & -0.464 & 0.003 & 0.009 & 0.002 & 0.006 \\
\hline 25th of Month & -18.823 & 0.230 & 0.257 & 0.007 & 0.001 & 0.000 & -0.007 \\
\hline Last Day of Year & -69.934 & -5.580 & 1.165 & 0.019 & 0.064 & 0.019 & -0.005 \\
\hline Last Day of Quarter & 46.993 & 6.739 & -3.087 & -0.007 & 0.035 & -0.006 & 0.009 \\
\hline Last 5 Days of Year & -70.441 & -10.953 & 0.153 & 0.003 & 0.020 & -0.007 & 0.000 \\
\hline Last 3 days of Quarter & 0.938 & 10.698 & 1.014 & 0.007 & 0.013 & 0.018 & 0.009 \\
\hline Target Fed Funds Rate (\%) & -0.190 & 0.034 & 0.004 & 0.000 & 0.000 & 0.000 & 0.000 \\
\hline Trend & -0.317 & 0.079 & 0.036 & 0 & 0.000 & 0.000 & 0.000 \\
\hline Constant & 2336.501 & 137.805 & 44.162 & 0.48 & 0.067 & 0.113 & 0.282 \\
\hline
\end{tabular}




\begin{tabular}{|r|rrrrr|}
\hline & & & & $\widehat{\Xi}$ & $\widehat{\Xi}$ \\
\hline Maint Day=1 & -0.350 & 0.059 & 0.004 & -0.004 & 0.051 \\
Maint Day=2 & 1.006 & 0.201 & 0.027 & 0.022 & -0.025 \\
Maint Day=3 & 0.048 & -0.013 & -0.003 & 0.013 & 0.071 \\
Maint Day=4 & -0.045 & 0.102 & 0.014 & -0.002 & -0.001 \\
Maint Day=6 & 0.286 & 0.003 & 0.003 & 0.036 & 0.005 \\
Maint Day=7 & 4.009 & 0.200 & 0.043 & 0.056 & 0.028 \\
Maint Day=8 & 1.218 & 0.090 & 0.004 & 0.013 & 0.031 \\
Maint Day=9 & 0.616 & 0.164 & 0.029 & 0.029 & -0.005 \\
Maint Day=10 & -1.070 & 0.395 & 0.065 & 0.062 & 0.062 \\
After 1 Day Holiday & -1.234 & -0.035 & -0.004 & -0.202 & 0.041 \\
After 3 Day Holiday & 4.584 & 0.046 & 0.011 & -0.075 & 0.053 \\
Before 1 Day Holiday & -1.162 & -0.179 & 0.017 & -0.063 & 0.000 \\
Before 3 Day Holiday & 3.723 & 0.272 & 0.041 & -0.019 & 0.012 \\
1st of Month & 10.112 & 0.226 & 0.054 & 0.051 & -0.028 \\
15th of Month & 4.912 & 0.113 & 0.046 & 0.103 & -0.035 \\
25th of Month & -6.051 & 0.067 & 0.004 & 0.048 & 0.155 \\
Last Day of Year & -17.893 & 0.080 & -0.021 & 0.223 & 0.349 \\
Last Day of Quarter & 9.963 & 0.034 & 0.026 & 0.084 & -0.147 \\
Last 5 Days of Year & -11.133 & -0.069 & -0.055 & -0.086 & -0.069 \\
Last 3 days of Quarter & -3.273 & 0.645 & 0.022 & 0.097 & 0.113 \\
Target Fed Funds Rate (\%) & -0.059 & 0.005 & 0.000 & 0.000 & 0.000 \\
Trend & -0.081 & 0.000 & 0.000 & 0.000 & 0.000 \\
Constant & 681.851 & 5.083 & 3.451 & 5.079 & 4.385 \\
\hline & & & & &
\end{tabular}

Table 8: Predicting network statistics using calendar dummies. Red indicates a p-value less than 0.001. Blue indicates a p-value betwen 0.001 and 0.01. Green indicates a p-value between 0.01 and 0.05 . The data used are April 1997 to December 2006.

\section{B Data}

Fedwire is the primary interbank payment system in the United States and is used to process large value and time critical payments between banks. Fedwire has more than 7,000 participants and processes, on average, 545, 000 payments worth $\$ 2.4$ trillion per day as of the fourth quarter of 2006 .

Since Fedwire transactions are not explicitly identified as federal funds loans, we identify transactions as candidates for overnight loans as transactions with value greater than or equal to $\$ 1$ million dollars and in round lots of $\$ 100,000$. We mark a transaction as a federal funds loan if there is a return transaction the next business day for the original amount plus interest within a range of plausible federal funds rates. We use 50 basis points above and below the daily maximum and minimum rates published by the Markets Group of the Federal Reserve Bank of New York as the range of acceptable interest ${ }^{18}$. This algorithm was introduced in Furfine (1999).

Demiralp et al. (2004) conduct a detailed analysis of the robustness of the Furfine algorithm. They allow for loans as low as $\$ 50,000$, in round lots of $\$ 50,000$, with rates that are in the range of 100 basis points above and below the maximum and minimum reported rates. They also force the rates to be in increments of 1 basis point or $\frac{1}{32}$ of a percent. Finally Demiralp et al. (2004) also allow for separate payment and interest return payments. Using these parameters, they report similar results as Furfine.

The algorithm cannot distinguish between federal funds loans and Eurodollar loans (although most Eurodollar transfers traditionally settle over CHIPS). This may not be a large issue, since Bartolini et al. (2005a) provides evidence that federal funds and Eurodollar loans are close substitutes.

The algorithm may also fail to identify certain overnight loans if these loans do not match our assumed characteristics. Most notably, we do not capture any fed funds loans that occur outside of Fedwire, such as transfers entirely on a broker's books or correspondent re-bookings. Data on the federal funds and Eurodollar loans of Euroclear in Bartolini et al. (2005b) confirm many of the assumptions of Furfine. The minimum

\footnotetext{
${ }^{18}$ The rates that the Markets Group reports are from a daily survey of the five largest federal funds brokers.
} 
loan was $\$ 50,000$, with only a handful-less than $0.1 \%$-under $\$ 1$ million. $94.3 \%$ of the loans were in round lots of $\$ 100,000$.

We use data from 2,454 business days from April 1, 1997 to December 29, 2006. 10 days of data were missing: December 22-23, 1997; March 1-2, 1999; April 20, 21, 28 and 29, 1999; and October 14-15, 1999. Additionally, we excluded 29 days for which the ratio of federal funds value to total Fedwire activity was more than 2.5 standard deviations from the mean: June 30, 1997; December 15 and 24, 1997; January 20, 1998; April 9-10, 1998; June 30, 1998; July 2, 1998; April 1-2, 1999; December 23-24, 1999; April 20-21, 2000; April 13, 2001;March 28-29, 2002; July 30, 2002; April 18, 2003; April 9, 2004; June 10, 2004; December 23-24, 2004; March 24, 2005; April 13-14, 2006; and May 11, 2006. This follows the practice of Demiralp et al. (2004), who note that these days are most likely the result of the algorithm misidentifying loans. Two additional days, with suspiciously low volumes were deleted. These were January 31, 2000 and July 2, 2001. Many of the days removed from consideration are Good Friday or the following Monday. In the end we are left with 2, 415 days of federal funds data. 


\section{European Central Bank Working Paper Series}

For a complete list of Working Papers published by the ECB, please visit the ECB's website (http://www.ecb.europa.eu).

929 "Real convergence in Central and Eastern European EU Member States: which role for exchange rate volatility?" by O. Arratibel, D. Furceri and R. Martin, September 2008.

930 "Sticky information Phillips curves: European evidence” by J. Döpke, J. Dovern, U. Fritsche and J. Slacalek, September 2008.

931 “International stock return comovements” by G. Bekaert, R. J. Hodrick and X. Zhang, September 2008.

932 "How does competition affect efficiency and soundness in banking? New empirical evidence" by K. Schaeck and M. Čihák, September 2008.

933 "Import price dynamics in major advanced economies and heterogeneity in exchange rate pass-through" by S. Dées, M. Burgert and N. Parent, September 2008.

934 “Bank mergers and lending relationships" by J. Montoriol-Garriga, September 2008.

935 "Fiscal policies, the current account and Ricardian equivalence" by C. Nickel and I. Vansteenkiste, September 2008.

936 “Sparse and stable Markowitz portfolios" by J. Brodie, I. Daubechies, C. De Mol, D. Giannone and I. Loris, September 2008.

937 "Should quarterly government finance statistics be used for fiscal surveillance in Europe?" by D. J. Pedregal and J. J. Pérez, September 2008.

938 "Channels of international risk-sharing: capital gains versus income flows" by T. Bracke and M. Schmitz, September 2008.

939 “An application of index numbers theory to interest rates” by J. Huerga and L. Steklacova, September 2008.

940 "The effect of durable goods and ICT on euro area productivity growth?" by J. Jalava and I. K. Kavonius, September 2008.

94I “The euro's influence upon trade: Rose effect versus border effect” by G. Cafiso, September 2008.

942 “Towards a monetary policy evaluation framework" by S. Adjemian, M. Darracq Pariès and S. Moyen, September 2008.

943 "The impact of financial position on investment: an analysis for non-financial corporations in the euro area" by C. Martinez-Carrascal and A. Ferrando, September 2008.

944 "The New Area-Wide Model of the euro area: a micro-founded open-economy model for forecasting and policy analysis" by K. Christoffel, G. Coenen and A. Warne, October 2008.

945 “Wage and price dynamics in Portugal” by C. Robalo Marques, October 2008.

946 “Macroeconomic adjustment to monetary union” by G. Fagan and V. Gaspar, October 2008.

947 "Foreign-currency bonds: currency choice and the role of uncovered and covered interest parity" by M. M. Habib and M. Joy, October 2008. 
948 "Clustering techniques applied to outlier detection of financial market series using a moving window filtering algorithm” by J. M. Puigvert Gutiérrez and J. Fortiana Gregori, October 2008.

949 “Short-term forecasts of euro area GDP growth” by E. Angelini, G. Camba-Méndez, D. Giannone, L. Reichlin and G. Rünstler, October 2008.

950 "Is forecasting with large models informative? Assessing the role of judgement in macroeconomic forecasts" by R. Mestre and P. McAdam, October 2008.

95I "Exchange rate pass-through in the global economy: the role of emerging market economies" by M. Bussière and T. Peltonen, October 2008.

952 "How successful is the G7 in managing exchange rates?" by M. Fratzscher, October 2008.

953 "Estimating and forecasting the euro area monthly national accounts from a dynamic factor model" by E. Angelini, M. Bańbura and G. Rünstler, October 2008.

954 "Fiscal policy responsiveness, persistence and discretion" by A. Afonso, L. Agnello and D. Furceri, October 2008.

955 "Monetary policy and stock market boom-bust cycles" by L. Christiano, C. llut, R. Motto and M. Rostagno, October 2008.

956 "The political economy under monetary union: has the euro made a difference?" by M. Fratzscher and L. Stracca, November 2008.

957 "Modeling autoregressive conditional skewness and kurtosis with multi-quantile CAViaR" by $\mathrm{H}$. White, T.-H. Kim, and S. Manganelli, November 2008.

958 "Oil exporters: in search of an external anchor” by M. M. Habib and J. Stráský, November 2008.

959 “What drives U.S. current account fluctuations?” by A. Barnett and R. Straub, November 2008.

960 “On implications of micro price data for macro models” by B. Maćkowiak and F. Smets, November 2008.

96I "Budgetary and external imbalances relationship: a panel data diagnostic" by A. Afonso and C. Rault, November 2008.

962 "Optimal monetary policy and the transmission of oil-supply shocks to the euro area under rational expectations" by S. Adjemian and M. Darracq Pariès, November 2008.

963 "Public and private sector wages: co-movement and causality" by A. Lamo, J. J. Pérez and L. Schuknecht, November 2008.

964 “Do firms provide wage insurance against shocks? Evidence from Hungary” by G. Kátay, November 2008.

965 “IMF lending and geopolitics” by J. Reynaud and J. Vauday, November 2008.

966 “Large Bayesian VARs” by M. Bańbura, D. Giannone and L. Reichlin, November 2008.

967 "Central bank misperceptions and the role of money in interest rate rules" by V. Wieland and G. W. Beck, November 2008.

968 “A value at risk analysis of credit default swaps” by B. Raunig and M. Scheicher, November 2008.

969 "Comparing and evaluating Bayesian predictive distributions of asset returns" by J. Geweke and G. Amisano, November 2008. 
970 "Responses to monetary policy shocks in the east and west of Europe" by M. Jarociński, November 2008.

97I “Interactions between private and public sector wages” by A. Afonso and P. Gomes, November 2008.

972 "Monetary policy and housing prices in an estimated DSGE for the US and the euro area" by M. Darracq Pariès and A. Notarpietro, November 2008.

973 "Do China and oil exporters influence major currency configurations?" by M. Fratzscher and A. Mehl, December 2008.

974 "Institutional features of wage bargaining in 23 European countries, the US and Japan” by P. Du Caju, E. Gautier, D. Momferatou and M. Ward-Warmedinger, December 2008.

975 "Early estimates of euro area real GDP growth: a bottom up approach from the production side" by E. Hahn and F. Skudelny, December 2008.

976 "The term structure of interest rates across frequencies" by K. Assenmacher-Wesche and S. Gerlach, December 2008.

977 "Predictions of short-term rates and the expectations hypothesis of the term structure of interest rates" by M. Guidolin and D. L. Thornton, December 2008.

978 "Measuring monetary policy expectations from financial market instruments" by M. Joyce, J. Relleen and S. Sorensen, December 2008.

979 "Futures contract rates as monetary policy forecasts" by G. Ferrero and A. Nobili, December 2008.

980 "Extracting market expectations from yield curves augmented by money market interest rates: the case of Japan" by T. Nagano and N. Baba, December 2008.

981 "Why the effective price for money exceeds the policy rate in the ECB tenders?” by T. Välimäki, December 2008.

982 “Modelling short-term Interest rate spreads in the euro money market?" by N. Cassola and C. Morana, December 2008.

983 "What explains the spread between the euro overnight rate and the ECB's policy rate?" by T. Linzert and S. Schmidt December 2008.

984 “The daily and policy-relevant liquidity effects” by D. L. Thornton, December 2008.

985 "Portuguese banks in the euro area market for daily funds" by L. Farinha and V. Gaspar, December 2008.

986 "The topology of the federal funds market" by M. L. Bech and E. Atalay, December 2008. 
